Algebraic $8 \mathcal{G}$ Geometric $\mathcal{T}$ opology

Volume 4 (2004) 721-755

Published: 11 September 2004

ATG

\title{
Peripheral separability and cusps of arithmetic hyperbolic orbifolds
}

\author{
D.B. MCREYNOLDS
}

\begin{abstract}
For $X=\mathbf{R}, \mathbf{C}$, or $\mathbf{H}$, it is well known that cusp cross-sections of finite volume $X$-hyperbolic $(n+1)$-orbifolds are flat $n$-orbifolds or almost flat orbifolds modelled on the $(2 n+1)$-dimensional Heisenberg group $\mathfrak{N}_{2 n+1}$ or the $(4 n+3)$-dimensional quaternionic Heisenberg group $\mathfrak{N}_{4 n+3}(\mathbf{H})$. We give a necessary and sufficient condition for such manifolds to be diffeomorphic to a cusp cross-section of an arithmetic $X$-hyperbolic $(n+1)$-orbifold.
\end{abstract}

A principal tool in the proof of this classification theorem is a subgroup separability result which may be of independent interest.

AMS Classification 57M50; 20G20

Keywords Borel subgroup, cusp cross-section, hyperbolic space, nil manifold, subgroup separability.

\section{Introduction}

\subsection{Main results}

A classical question in topology is whether a compact manifold bounds. Hamrick and Royster [15] showed that every flat manifold bounds, and it was conjectured in [11] that any almost flat manifold bounds (for some progress on this see [24] and [32]). In [11, Farrell and Zdravkovska made a stronger geometric conjecture:

\section{Conjecture 1.1}

(a) If $M^{n}$ is a flat Riemannian manifold, then $M^{n}=\partial W^{n+1}$ where $W \backslash \partial W$ supports a complete hyperbolic structure with finite volume.

(b) If $M^{n}$ supports an almost flat structure, then $M^{n}=\partial W^{n+1}$, where $W \backslash \partial W$ supports a complete Riemannian metric with finite volume of whose sectional curvatures are negative. 
We say that a flat manifold $M^{n}$ geometrically bounds if (a) in Conjecture 1.1 holds. Long and Reid [19] showed that (a) is false by proving that for a flat $(4 n-1)$-manifold to geometrically bound the $\eta$-invariant is an integer. Furthermore, flat 3-manifolds with nonintegral $\eta$-invariant are easily constructed using [25]. Equivalently, this result of Long and Reid shows that some flat manifolds cannot be diffeomorphic to a cusp cross-section of a 1-cusped, finite volume real hyperbolic 4-manifold. On the other hand, Long and Reid showed [20] that every flat $n$-manifold is diffeomorphic to a cusp cross-section of an arithmetic real hyperbolic $(n+1)$-orbifold.

For $X=\mathbf{R}, \mathbf{C}$, or $\mathbf{H}$, cusp cross-sections of finite volume $X$-hyperbolic $(n+1)$-orbifolds are flat $n$-manifolds or almost flat orbifolds modelled on the $(2 n+1)$-dimensional Heisenberg group $\mathfrak{N}_{2 n+1}$ or the $(4 n+3)$-dimensional quaternionic Heisenberg group $\mathfrak{N}_{4 n+3}(\mathbf{H})$. The first main result of this article shows that the result of Long and Reid in 20] does not generalize to the complex or quaternionic settings. Namely, (see $\S 2$ for definitions):

\section{Theorem 1.2}

(a) For every $n \geq 2$, there exist infinite families of closed almost flat $(2 n+1)-$ manifolds modelled on $\mathfrak{N}_{2 n+1}$ which are not diffeomorphic to a cusp crosssection of any arithmetic complex hyperbolic $(n+1)$-orbifold.

(b) For every $n \geq 1$, there exist infinite families of closed almost flat $(4 n+3)-$ manifolds modelled on $\mathfrak{N}_{4 n+3}(\mathbf{H})$ which are not diffeomorphic to a cusp cross-section of any finite volume quaternionic hyperbolic $(n+1)$-orbifold.

Since all lattices in the isometry group of quaternionic hyperbolic space are arithmetic (see [7]), we drop the arithmeticity assumption in (b).

In order to give a complete classification of cusp cross-sections of arithmetic hyperbolic lattices, we require certain subgroup separability results. Recall that if $G$ is a group, $H<G$ and $g \in G \backslash H$, we say $H$ and $g$ are separated if there exists a subgroup $K$ of finite index in $G$ which contains $H$ but not $g$. We say that $H$ is separable in $G$ or $G$ is $H$-separable, if every $g \in G \backslash H$ and $H$ can be separated. We say that $G$ is $L E R F$ (locally extendable residually finite) if every finitely generated subgroup is separable.

We defer the statement of our second main result until $\S 3$ (see Theorem 3.1) as it requires the language of algebraic groups. Instead we state the result specialized to the rank-1 setting. For the statement, let $Y=\mathbf{H}_{\mathbf{R}}^{n}, \mathbf{H}_{\mathbf{C}}^{n}, \mathbf{H}_{\mathbf{H}}^{n}$ or $\mathbf{H}_{\mathbf{O}}^{2}$. 
Theorem 1.3 (Stabilizer subgroup separability theorem) Let $\Lambda$ be an arithmetic lattice in $\operatorname{Isom}(Y)$ and $v \in \partial Y$. Then every subgroup of $\Lambda \cap \operatorname{Stab}(v)$ is separable in $\Lambda$.

One well known application of subgroup separability is the lifting of an immersion to an embedding in a finite cover (see [18, [14, or [21, p. 176]). In the rank-1 setting, we have (see Theorem 3.12 for a more general result):

Theorem 1.4 Let $\rho: N \longrightarrow M$ be a $\pi_{1}$-injective immersion of an almost flat manifold $N$ modelled in $\mathfrak{N}_{\ell n-1}(X)$ into an arithmetic $X$-hyperbolic $m-$ orbifold. Then there exists a finite cover $\psi: M^{\prime} \longrightarrow M$ such that $\rho$ lifts to an embedding.

A geometric corollary of Theorem 1.3 of particular interest to us is:

\section{Theorem 1.5}

(a) A flat n-manifold is diffeomorphic to a cusp cross-section of an arithmetic real hyperbolic $(n+1)$-orbifold if and only if $\pi_{1}\left(M^{n}\right)$ injects into an arithmetic real hyperbolic $(n+1)$-lattice.

(b) An almost flat $(2 n+1)$-manifold $M^{2 n+1}$ modelled on $\mathfrak{N}_{2 n+1}$ is diffeomorphic to a cusp cross-section of an arithmetic complex hyperbolic $(n+1)-$ orbifold if and only if $\pi_{1}\left(M^{2 n+1}\right)$ injects into an arithmetic complex hyperbolic $(n+1)$-lattice.

(c) An almost flat $(4 n+3)$-manifold $M^{4 n+3}$ modelled on $\mathfrak{N}_{4 n+3}(\mathbf{H})$ is diffeomorphic to a cusp cross-section of a quaternionic hyperbolic $(n+1)-$ orbifold if and only if $\pi_{1}\left(M^{2 n+1}\right)$ injects into a quaternionic hyperbolic $(n+1)$-lattice.

(d) An almost flat 15-manifold $M^{15}$ modelled on $\mathfrak{N}_{15}(\mathbf{O})$ is diffeomorphic to a cusp cross-section of an octonionic hyperbolic 16-orbifold if and only if $\pi_{1}\left(M^{15}\right)$ injects into an octonionic hyperbolic 16-lattice.

Theorem 1.5 reduces the classification of cusp-cross sections of arithmetic $X-$ hyperbolic $n$-orbifolds to the construction of faithful representations of almost flat manifold groups into lattices. We postpone stating the classification until $\S 5$ (see Theorem 5.4) as it requires additional terminology. However, one interesting special case which we state here is (see $\S 7$ for a proof):

Corollary 1.6 Every nil 3-manifold is diffeomorphic to a cusp cross-section of an arithmetic complex hyperbolic 2-orbifold. 
The rest of the paper is organized as follows. We establish notation and collect the preliminary material in $\S 2$ needed in the sequel. Our main separability results are established in $\S 3$ and $\S 4$ along with some algebraic and geometric corollaries. In $\S 5$ we classify cusp cross-section of arithmetic hyperbolic orbifolds and give the families of Theorem 1.2 in $\S 6$. We conclude this article with a detailed treatment of the nil $3-$ manifold case in $\S 7$.

\subsection{Acknowledgments}

I would like to thank my advisor Alan Reid for all his help. I am indebted to Daniel Allcock for several helpful suggestions, most notably the use of central products. In addition, I would like to thank Karel Dekimpe, Yoshinobu Kamishima, Richard Kent, and Richard Schwartz for conversations on this work. Finally, I would like to thank the referee for several valuable comments and for informing me of Proposition 3.8

\section{Preliminary material}

In this section, we rapidly develop the material needed in the sequel.

\section{$2.1 \quad X$-hyperbolic $n$-space}

For a general reference on this material, see [5, II.10]. In all that follows, we let $X=\mathbf{R}, \mathbf{C}$, or $\mathbf{H}$ and $\ell=\operatorname{dim}_{\mathbf{R}} X$.

Equip $X^{n+1}$ with a Hermitian form $H$ of signature $(n, 1)$. We define $X-$ hyperbolic $n$-space to be the (left) $X$-projectivization of the $H$-negative vectors with the Bergmann metric associated to $H$. We denote $X$-hyperbolic $n$-space together with this metric by $\mathbf{H}_{X}^{n}$ and say that $\mathbf{H}_{X}^{n}$ is modelled on $H$ or call $H$ a model form.

The boundary of $\mathbf{H}_{X}^{n}$ in $P X^{n+1}$ is the $X$-projectivization of the $H$-null vectors. We denote this set by $\partial \mathbf{H}_{X}^{n}$, which is topologically just $S^{\ell n}$ (see [5] p. 265]) and call the elements of the boundary light-like vectors. 


\subsection{The isometry group and lattices}

The isometry group of $\mathbf{H}_{X}^{n}$ is denoted by $\operatorname{Isom}\left(\mathbf{H}_{X}^{n}\right)$. In each setting, $\operatorname{Isom}\left(\mathbf{H}_{X}^{n}\right)$ is locally isomorphic to $\mathrm{U}(H)$. Specifically,

$$
\operatorname{Isom}\left(\mathbf{H}_{X}^{n}\right)= \begin{cases}\left\langle\mathrm{PU}(H)_{0}, \iota\right\rangle, & X=\mathbf{R}, \mathbf{C} \\ \mathrm{PU}(H), & X=\mathbf{H},\end{cases}
$$

where $\iota$ is an involution induced by inversion in the real case and complex conjugation in the complex case. The usual trichotomy for isometries holds in $\operatorname{Isom}\left(\mathbf{H}_{X}^{n}\right)$ (see [28, p. 180-185], 12, p. 203], 17]). Specifically, every (nontrivial) isometry is either elliptic, parabolic, or loxodromic.

We say that $\Gamma<\operatorname{Isom}\left(\mathbf{H}_{X}^{n}\right)$ is a lattice if $\Gamma$ is a discrete subgroup and $\mathbf{H}_{X}^{n} / \Gamma$ has finite volume. In this case, $M=\mathbf{H}_{X}^{n} / \Gamma$ is called an $X$-hyperbolic $n$ orbifold. That finite volume manifolds exist, both compact and noncompact, was established by Borel [3].

The spaces constructed in this way yield every locally symmetric space of rank1 except for those modelled on the exceptional Cayley hyperbolic plane $\mathbf{H}_{\mathbf{O}}^{2}$. We shall only make use of the fact that $\operatorname{Isom}\left(\mathbf{H}_{\mathbf{O}}^{2}\right)$ has a faithful linear representation and refer the reader to [1] for more on the Cayley hyperbolic plane.

\subsection{The Heisenberg group and its quaternionic analog}

In the next few subsections, we introduce the $X$-Heisenberg group and its automorphism group. See [12 for the complex case and [17] for the quaternionic case. A thorough treatment of this topic can be found in [24].

Let $\langle\cdot, \cdot\rangle$ denote the standard Hermitian product on $X^{n}$ and let $\omega=\operatorname{Im}\langle\cdot, \cdot\rangle$ be the associated hyper-symplectic form. The $X$-Heisenberg group $\mathfrak{N}_{\ell n-1}(X)$ is defined to be the topological space $X^{n-1} \times \operatorname{Im} X$ together with the group structure

$$
\left(\xi_{1}, t_{1}\right) \cdot\left(\xi_{2}, t_{2}\right) \stackrel{\text { def }}{=}\left(\xi_{1}+\xi_{2}, t_{1}+t_{2}+2 \omega\left(\xi_{1}, \xi_{2}\right)\right) .
$$

The Lie group $\mathfrak{N}_{\ell n-1}(X)$ is simply connected and connected. Moreove, the group $\mathfrak{N}_{\ell n-1}(X)$ is nilpotent of step size two in the case $X \neq \mathbf{R}$ and abelian in the case $X=\mathbf{R}$. The center (in the nonabelian cases) is the commutator subgroup, which can be identified with $\{0\} \times \operatorname{Im} X$. 


\subsection{Automorphisms of the $X$-Heisenberg group}

The automorphism group of the $X$-Heisenberg group Aut $\left(\mathfrak{N}_{\ell n-1}(X)\right)$ splits as $\operatorname{Inn}\left(\mathfrak{N}_{\ell n-1}\right) \rtimes \operatorname{Out}\left(\mathfrak{N}_{\ell n-1}\right)$. The inner automorphism group can be identified with the vectors in $X^{n-1}$ which are $\omega$-nondegenerate together with the zero vector. In the real case, this set is just $\{0\}$, while in the other two cases, this is the whole of $X^{n-1}$.

The outer automorphism group is comprised of three types of automorphisms. The first type of automorphism is a symplectic rotation given by $S(\xi, t)=(S \xi, t)$ for $S \in \operatorname{Sp}(\omega)$. The second type of automorphism is a Heisenberg dilation given by $d(\xi, t)=\left(d \xi, d^{2} t\right)$ for $d \in \mathbf{R}^{\times}$. Finally, we have $X$-scalar conjugation given by $\zeta(\xi, t)=\left(\zeta^{-1} \xi \zeta, \zeta^{-1} t \zeta\right)$ for $\zeta \in X^{\times}$. The outer automorphism group is generated by these three automorphisms. In summary, we have

$$
\operatorname{Out}\left(\mathfrak{N}_{\ell n-1}(X)\right)= \begin{cases}\operatorname{GL}(n-1 ; \mathbf{R}), & X=\mathbf{R} \\ \operatorname{Sp}(2 n-2) \times \mathbf{R}^{\times}, & X=\mathbf{C} \\ \operatorname{Sp}(\omega) \times \mathbf{R}^{\times} \times \mathbf{H}^{\times}, & X=\mathbf{H} .\end{cases}
$$

\subsection{Maximal compact subgroups}

Our primary concern is with maximal compact subgroups $\operatorname{Aut}\left(\mathfrak{N}_{\ell n-1}\right)$. The maximal compact subgroups are of the form

$$
M(X)= \begin{cases}\mathrm{O}\left(B_{M(X)}\right), & X=\mathbf{R} \\ \left\langle\mathrm{U}\left(H_{M(X)}\right), \iota\right\rangle, & X=\mathbf{C} \\ \mathrm{U}\left(H_{M(X)}\right) \times S, & X=\mathbf{H},\end{cases}
$$

where $B_{M(X)}$ is a symmetric, positive definite bilinear form, $H_{M(X)}$ is a signature $(n-1,0)$ Hermitian form with $\operatorname{Im} H_{M(X)}=\omega$, and $S$ is the unit sphere in $\mathbf{H}$ (equipped possibly with a nonstandard quaternionic structure). Since the maximal compact subgroups are conjugate, each $M(X)$ is conjugate to

$$
M_{s}(X)= \begin{cases}\mathrm{O}(n-1), & X=\mathbf{R} \\ \langle\mathrm{U}(n-1), \iota\rangle, & X=\mathbf{C} \\ \mathrm{Sp}(n-1) \times \mathrm{SO}(3), & X=\mathbf{H} .\end{cases}
$$

For a given maximal compact subgroup $M$, we call the group $\mathfrak{N}_{\ell n-1}(X) \rtimes M$ a unitary affine group and denote this group by $U_{M}(n-1 ; X)$. We call the group $\mathfrak{N}_{\ell n-1}(X) \rtimes\left(M(X) \times \mathbf{R}^{+}\right)$an $X$-Heisenberg similarity group and denote this group by $S_{M}(n-1 ; X)$. Finally, we call the group $\mathfrak{N}_{\ell n-1}(X) \rtimes \operatorname{Aut}\left(\mathfrak{N}_{\ell n-1}(X)\right)$ the $X$-Heisenberg affine group and denote it by $\operatorname{Aff}\left(\mathfrak{N}_{\ell n-1}(X)\right)$. 


\subsection{Almost crystallographic groups modelled on the $X-$ Heisenberg group}

In this subsection, we introduce almost crystallographic groups modelled on the $X$-Heisenberg group. We refer the reader to [9] or [27, Chapter II and VIII] for a general treatment on discrete subgroups in nilpotent Lie groups.

By an almost crystallographic group or $A C$-group modelled on $\mathfrak{N}_{\ell n-1}(X)$, we mean a discrete subgroup $\Gamma<\operatorname{Aff}\left(\mathfrak{N}_{\ell n-1}\right)(X)$ such that $\mathfrak{N}_{\ell n-1}(X) / \Gamma$ is compact and $\Gamma \cap \mathfrak{N}_{\ell n-1}(X)$ is a finite index subgroup of $\Gamma$. When $\Gamma$ is torsion free, we say that $\Gamma$ is an almost Bieberbach group or AB-group modelled on $\mathfrak{N}_{\ell n-1}(X)$. Every AC-group modelled on $\mathfrak{N}_{\ell n-1}(X)$ is determined by the short exact sequence

$$
1 \longrightarrow L \longrightarrow \Gamma \longrightarrow \theta \longrightarrow 1
$$

where $L=\Gamma \cap \mathfrak{N}_{\ell n-1}(X)$ and $|\theta|<\infty$. We call $L$ the Fitting subgroup of $\Gamma$ and $\theta$ the holonomy group of $\Gamma$.

It is well known (see 9 , Ch. 3]) that the above exact sequence induces an injective homomorphism $\varphi: \theta \longrightarrow \operatorname{Out}\left(\mathfrak{N}_{\ell n-1}(X)\right)<\operatorname{Aut}\left(\mathfrak{N}_{\ell n-1}(X)\right)$ which we call the holonomy representation of $\theta$. Since $\theta$ is finite, this is conjugate into a representation $\varphi: \theta \longrightarrow M(X)$ for any $M(X)$. This yields a faithful representation $\rho: \Gamma \longrightarrow U_{M}(n-1 ; X)$ for any $M(X)$.

\subsection{Almost flat manifolds}

Let $\left(M^{n}, g\right)$ be a complete Riemannian manifold. We let $d=d(g), c^{-}(g)$ and $c^{+}(g)$ denote the diameter of $M$ and the lower and upper bounds of the sectional curvature of $M$, respectively, and set $c(g)$ to be the maximum of $\left|c^{+}\right|$ and $\left|c^{-}\right|$. We say that $M$ is almost flat if there exists a family of complete Riemannian metrics $g_{j}$ on $M$ such that

$$
\lim _{j \longrightarrow \infty} d\left(g_{j}\right)^{2} c\left(g_{j}\right)=0 .
$$

Gromov 13. proved that every compact almost flat manifold is of the form $N / \Gamma$, where $N$ is a connected, simply connected nilpotent Lie group and $\Gamma$ is an AB-group modelled on $N$.

Of importance to us is some of the generalized Bieberbach theorem (see [9]). 
Theorem 2.1 (Generalized Bieberbach theorem)

(a) Let $M$ be an almost flat manifold with universal cover $\mathfrak{N}_{\ell n-1}$. Then there exists a faithful representation $\varphi: \pi_{1}(M) \longrightarrow \operatorname{Aff}\left(\mathfrak{N}_{\ell n-1}(X)\right)$ such that $\varphi\left(\pi_{1}(M)\right)$ is an AB-group.

(b) $\quad M=\mathfrak{N}_{\ell n-1} / \Gamma$ and $M^{\prime}=\mathfrak{N}_{\ell n-1} / \Gamma^{\prime}$ are diffeomorphic if and only if there exists $\alpha \in \operatorname{Aff}\left(\mathfrak{N}_{\ell n-1}\right)$ such that

$$
\Gamma^{\prime}=\alpha^{-1} \Gamma \alpha .
$$

In the remainder of this article we refer to compact almost flat manifolds as infranil manifolds modelled on $N$, where $N$ is the connected, simply connected nilpotent cover. In the event the fundamental group is a lattice in $N$, we call such manifolds nil manifolds modelled on $N$.

\subsection{Maximal peripheral subgroups, stabilizer groups, and cusps}

For a lattice $\Lambda<\operatorname{Isom}\left(\mathbf{H}_{X}^{n}\right)$ with cusp at $v$, we define the maximal peripheral subgroup of $\Lambda$ at $v$ to be the subgroup $\triangle_{v}(\Lambda)=\operatorname{Stab}(v) \cap \Lambda$. This is the subgroup generated by the parabolic and elliptic isometries of $\Lambda$ fixing $v$. By the Kazhdan-Margulis theorem (this is sometimes called Margulis' lemma; see [27, Chapter XI]), $\triangle_{v}(\Lambda)$ is virtually nilpotent. Specifically, the maximal nilpotent subgroup of $\triangle_{v}(\Lambda)$ is given by $L=\triangle_{v}(\Lambda) \cap N$, where $N$ is isomorphic to $\mathfrak{N}_{\ell n-1}(X)$. Moreover, the Kazhdan-Margulis theorem allows us to select a horosphere $\mathcal{H}$ such that $\mathcal{H} / \triangle_{v}(\Lambda)$ is embedded in $\mathbf{H}_{X}^{n} / \Lambda$. In this case, we call $\mathcal{H} / \triangle_{v}(\Lambda)$ a cusp cross-section of the cusp at $v$. Often when $v$ is unimportant, we simply write $\triangle(\Lambda)$.

More generally, for any $v \in \partial \mathbf{H}_{X}^{n}$, we define $\triangle_{v}(\Lambda)=\Lambda \cap \operatorname{Stab}(v)$ and call this subgroup the stabilizer group of $\Lambda$ at $v$. There are three possibilities:

(1) $\triangle_{v}(\Lambda)$ is finite.

(2) $\triangle_{v}(\Lambda)$ is virtually cyclic with cyclic subgroup generated by a loxodromic isometry.

(3) $\triangle_{v}(\Lambda)$ is an AC-group modelled on $\mathfrak{N}_{\ell n-1}(X)$.

\subsection{Iwasawa decompositions of the isometry group}

For the isometry group of $X$-hyperbolic $n$-space, we can decompose $\operatorname{Isom}\left(\mathbf{H}_{X}^{n}\right)$ as $K A N$ via the Iwasawa decomposition (see [5. p. 311-313]). The factor $N$ is 
isomorphic to the $X$-Heisenberg group $\mathfrak{N}_{\ell n-1}(X)$ and all isomorphisms arise in the following fashion. Let $H$ be a model Hermitian form for $X$-hyperbolic $n$-space and $V_{\infty}$ be the $H$-orthogonal complement of $v_{0}$ and $v_{\infty}$, a pair of $X-$ linearly independent $H$-null vectors in $X^{n+1}$. For a maximal compact group $M(X)$ with associated Hermitian form $H_{M(X)}$, let

$$
\psi:\left(X^{n-1}, H_{M(X)}\right) \longrightarrow\left(V_{\infty}, H_{\mid V_{\infty}}\right)
$$

be any isometric $X$-isomorphism. This induces a map $\eta: X^{n-1} \longrightarrow N$ defined by $\eta(\xi)=\exp \left(\psi(\xi) v_{\infty}^{*}-v_{\infty} \psi(\xi)^{*}\right)$, where $x y^{*}(\cdot)=H(\cdot, y) x$ is the Hermitian outer pairing of $x$ and $y$ with respect to the Hermitian form $H$. This extends to all of $\mathfrak{N}_{\ell n-1}(X)$ as these elements generate $\mathfrak{N}_{\ell n-1}(X)$. In fact, this extends to $\eta: S_{M}(n-1 ; X) \longrightarrow \operatorname{Isom}\left(\mathbf{H}_{X}^{n}\right)$. Since these isometries preserve $v_{\infty}$, this yields $\eta\left(S_{M}(n-1 ; X)\right)=\operatorname{Stab}\left(v_{\infty}\right)$.

\subsection{Algebraic groups}

As we use the language of algebraic groups throughout this paper, in this subsection we review some of the basic material. See [4] or [26, Ch. 2].

In the remainder of this article, all fields are assumed to be algebraic number fields unless stated otherwise.

By a linear algebraic group we mean a subgroup of $\mathrm{GL}(n ; \mathbf{C})$ which is closed in the Zariski topology. We say that $G$ is $k$-algebraic when there is a generating set of $k$-polynomials for $\mathfrak{a}_{G}$, the ideal vanishing on $G$. For any subring $R \subset \mathbf{C}$, we define the $R$-points of $G$ to be the subgroup $G \cap \mathrm{GL}(n ; R)$. We denote the $R$-points of $G$ by $G_{R}$.

A Borel subgroup of $G$ is a maximal, connected solvable subgroup of $G$. Borel subgroups of $G$ are conjugate in $G$ and conjugate into the subgroup of upper triangular matrices. If $G$ is $k$-algebraic, then $B$ will be $k^{\prime}$-algebraic for some finite extension $k^{\prime}$ of $k$.

A maximal algebraic torus $T$ of $G$ is a maximal diagonalizable algebraic subgroup. If $k$ is the field of definition for $G$, then the splitting field $k^{\prime}$ for $T$ is a finite extension of $k$. This is the smallest field for which $T$ can be diagonalized. In particular, $T$ will be a $k^{\prime}$-algebraic group. We say that $U<G$ is unipotent if $U$ is conjugate to a subgroup of the upper triangular matrices with ones along the diagonal. Maximal unipotent subgroups $U$ are connected, nilpotent, algebraic subgroups and if $G$ is $k$-algebraic, $U$ is $k^{\prime}$-algebraic for some finite extension $k^{\prime}$ of $k$. We note that every maximal torus $T$ or maximal unipotent subgroup $U$ in $G$ is contained in a Borel subgroup (see [4, Cor. 11.3]). 
Finally, we require the following lemma in the sequel and refer the reader to [27. Cor. 10.14] for a proof. In the statement, $\mathcal{O}_{k}$ denotes the ring of algebraic integers in the number field $k$ (see [36]).

Lemma 2.2 Let $f: G \longrightarrow G^{\prime}$ be a $k$-homomorphism of $k$-algebraic groups. If $\Gamma<G_{k}$ is commensurable with $G_{\mathcal{O}_{k}}$, then there exists $\Gamma^{\prime}<G_{k}^{\prime}$, commensurable with $G_{\mathcal{O}_{k}}^{\prime}$ such that $f(\Gamma)<\Gamma^{\prime}$.

\section{Borel subgroup separability theorem}

This section is devoted to proving the following result.

Theorem 3.1 (Borel subgroup separability theorem) Let $G$ be a connected $k$-algebraic group and $B$ a Borel subgroup of $G$. Then any subgroup of $B_{\mathcal{O}_{k}}$ is separable in $G_{\mathcal{O}_{k}}$.

Before embarking upon the proof, we record some facts that will be needed. We begin with the following lemma (see [20]).

Lemma 3.2 Let $G$ be a group and $H<K<G$. If $H$ is separable in $G$ and $[K: H]<\infty$, then $K$ is separable in $G$.

Lemma 3.3 Let $G$ be a group and assume that $H, L<G$ are separable in $G$. Then $H \cap L$ is separable in $G$.

Proof Let $\gamma \in G \backslash(H \cap L)$ and assume that $\gamma \notin H$. Since $H$ is separable in $G$, there exists a finite index subgroup $K<G$ with $H<K$ and $\gamma \notin K$. As $H \cap L<H, K$ separates $\gamma$ and $H \cap L$, as needed. For the alternative, an identical argument is made.

Lemma 3.4 Let $G$ be a group, $G_{0}$ a subgroup of finite index, and $H$ a subgroup. $H$ is separable in $G$ if and only if $\left(G_{0} \cap H\right)$ is separable in $G_{0}$.

Proof The direct implication follows immediately from Lemma 3.3. since $H \cap G_{0}$ is separable in the larger group $G$. For the reverse implication, to show that $H$ is separable in $G$, by Lemma 3.2 it suffices to show that $G_{0} \cap H$ is separable in $G$. For $g \in G \backslash\left(G_{0} \cap H\right)$, there are two cases to consider. If $g \notin G_{0}$, then $G_{0}$ separates $G_{0} \cap H$ and $g$. Otherwise, if $g \in G_{0}$, since $G_{0} \cap H$ is separable in $G_{0}$, there exists a finite index subgroup $K<G_{0}$ such that $G_{0} \cap H<K$ and $g \notin K$. Since $\left[G: G_{0}\right]<\infty, K$ is the desired finite index subgroup of $G$ separating $G_{0} \cap H$ and $g$. 
The separability of Borel subgroups relies on the following result of Chahal [6] which establishes the congruence subgroup property for solvable algebraic groups defined over number fields. Before we state the result, we recall the definition of congruence kernels and reduction homomorphisms.

For each ideal $\mathfrak{p}<\mathcal{O}_{k}$, we can define the homomorphism (reduction modulo $\mathfrak{p}$ ) $r_{\mathfrak{p}}: H_{\mathcal{O}_{k}} \longrightarrow \mathrm{GL}\left(m ; \mathcal{O}_{k} / \mathfrak{p}\right)$ by $r_{\mathfrak{p}}(\gamma)=\left(\gamma_{i j} \bmod \mathfrak{p}\right)_{i j}$. By a congruence kernel we mean a subgroup ker $r_{\mathfrak{p}}$, for some (nontrivial) ideal $\mathfrak{p}<\mathcal{O}_{k}$ and denote this subgroup by $K_{H, \mathfrak{p}}$.

Theorem 3.5 Let $H$ be a solvable $k$-algebraic group. Then every finite index subgroup of $H_{\mathcal{O}_{k}}$ contains a congruence kernel.

\subsection{The proof of Theorem 3.1}

For the proof of Theorem 3.1 recall that $G$ is a connected $k$-algebraic group with a Borel subgroup $B$ defined over $k^{\prime}$. The strategy for the proof is as follows. If $B$ is defined over $k$ ( $G$ is $k$-split), the proof reduces to proving that $B_{\mathcal{O}_{k}}$ is separable in $G_{\mathcal{O}_{k}}$. For once this has been established, to separate a subgroup of $B_{\mathcal{O}_{k}}$ in $G_{\mathcal{O}_{k}}$, it suffices to separate the subgroup in $B_{\mathcal{O}_{k}}$. The latter is achieved by appealing to a theorem of Mal'cev. In the non-split case when $k^{\prime}$ is not contained in $k$, we enlarge our field to the composite field of $k$ and $k^{\prime}$ and appeal to the split case. In the remainder of this subsection, we give the details.

The following two lemmas comprise the key steps in the proof of Theorem 3.1

Lemma 3.6 Let $G$ be a connected $k$-algebraic group and $B$ a $k$-defined Borel subgroup of $G$. If $B_{\mathcal{O}_{k}}$ is separable in $G_{\mathcal{O}_{k}}$, then every subgroup of $B_{\mathcal{O}_{k}}$ is separable in $G_{\mathcal{O}_{k}}$.

Lemma 3.7 Let $G$ be a connected $k$-algebraic group and $B$ a $k$-defined Borel subgroup of $G$. Then $B_{\mathcal{O}_{k}}$ is separable in $G_{\mathcal{O}_{k}}$.

Assuming these lemmas, we prove Theorem 3.1

Proof of Theorem 3.1 The proof breaks into two cases, depending on whether or not $k^{\prime} \subset k$.

Case $1 k^{\prime} \subset k$ 
Since $k^{\prime} \subset k, B$ is a $k$-defined Borel subgroup of $G$. Therefore by Lemma 3.7 $B_{\mathcal{O}_{k}}$ is separable in $G_{\mathcal{O}_{k}}$. Thus by Lemma [3.6 every subgroup of $B_{\mathcal{O}_{k}}$ is separable in $G_{\mathcal{O}_{k}}$, as desired.

Case $2 k^{\prime}$ is not contained in $k$

In this case, let $\widehat{k}$ denote the composite of $k$ and $k^{\prime}$. Then $G$ is a $\widehat{k}$-algebraic group and $B$ is a $\widehat{k}$-defined Borel subgroup. Therefore by Lemma 3.7 $B_{\mathcal{O}_{\widehat{k}}}$ is separable in $G_{\mathcal{O}_{\widehat{k}}}$. Thus by Lemma 3.6. every subgroup of $B_{\mathcal{O}_{\widehat{k}}}$ is separable in $G_{\mathcal{O}_{\widehat{k}}}$. Since $k \subset \widehat{k}, B_{\mathcal{O}_{k}} \subset B_{\mathcal{O}_{\widehat{k}}}$ and so every subgroup of $B_{\mathcal{O}_{k}}$ is separable in $G_{\mathcal{O}_{\widehat{k}}}$. Thus every subgroup of $B_{\mathcal{O}_{k}}$ is separable in the smaller group $G_{\mathcal{O}_{k}}$.

We are now left with the task of verifying Lemma 3.6 and Lemma 3.7.

Proof of Lemma 3.6 Let $S<B_{\mathcal{O}_{k}}$ be a subgroup. For $\gamma \in G_{\mathcal{O}_{k}} \backslash S$, there are two cases to consider. First, if $\gamma \notin B_{\mathcal{O}_{k}}$, then by the separability of $B_{\mathcal{O}_{k}}$ we can find a finite index subgroup $K<G_{\mathcal{O}_{k}}$ such that $S<B_{\mathcal{O}_{k}}<K$ and $\gamma \notin K$. If $\gamma \in B_{\mathcal{O}_{k}}$ we argue as follows. Since $B_{\mathcal{O}_{k}}$ is polycyclic (27, p. 53] or [34. p. 196]) it is LERF by [22]. Therefore there exists a finite index subgroup $K_{B}<B_{\mathcal{O}_{k}}$ such that $S<K_{B}$ and $\gamma \notin K_{B}$. By Theorem 3.5, $B_{\mathcal{O}_{k}}$ has the congruence subgroup property. Thus there exists a congruence kernel $K_{B, \mathfrak{p}}$ of $B_{\mathcal{O}_{k}}$ with $K_{B, \mathfrak{p}}<K_{B}$. As $K_{B, \mathfrak{p}}$ is the intersection of $B_{\mathcal{O}_{k}}$ with the congruence kernel $K_{G, \mathfrak{p}}$ of $G_{\mathcal{O}_{k}}$, by Lemma 3.3, $K_{B, \mathfrak{p}}$ is separable in $G_{\mathcal{O}_{k}}$. By Lemma 3.2. $K_{B}$ is separable in $G_{\mathcal{O}_{k}}$, since $\left[K_{B}: K_{B, \mathfrak{p}}\right]<\infty$. Consequently, we can find a finite index subgroup $K<G_{\mathcal{O}_{k}}$ such that $S<K_{B}<K$ and $\gamma \notin K$. Therefore $S$ and $\gamma$ are separated in $G_{\mathcal{O}_{k}}$.

The proof of Lemma 3.7 follows from a more general result established in 2 (see also [23]):

Proposition 3.8 Let $H$ be an algebraic group in a linear algebraic group $G$ and $\Gamma$ a finitely generated subgroup of $G$. Then $H \cap \Gamma$ is separable in $\Gamma$.

Lemma 3.7 follows from Proposition 3.8 by setting $H=B$ and $\Gamma=G_{\mathcal{O}_{k}}$.

The proof of Theorem 3.1 works in greater generality. Specifically,

Corollary 3.9 Let $G$ be a connected $k$-algebraic group and $N$ a $k^{\prime}$-algebraic subgroup with $k \subset k^{\prime}$. If $N_{\mathcal{O}_{k^{\prime}}}$ has the congruence subgroup property, then a finitely generated subgroup $L$ of $N_{\mathcal{O}_{k}}$ is separable in $G_{\mathcal{O}_{k}}$ if and only if $L$ is separable in $N_{\mathcal{O}_{k}}$. 


\subsection{Corollaries to Theorem 3.1}

In this subsection, we state a few corollaries to Theorem 3.1 pertaining to general algebraic groups.

Our first corollary shows that the conclusions of Theorem 3.1 hold for any subgroup of $G$ commensurable with $G_{\mathcal{O}_{k}}$. We call such subgroups $k$-arithmetic subgroups.

Corollary 3.10 Let $G$ be a connected $k$-algebraic group, $\Lambda$ a $k$-arithmetic subgroup in $G$, and $B$ a Borel subgroup of $G$. Then every subgroup of $\Lambda \cap B$ is separable in $\Lambda$.

Proof For a subgroup $S<B \cap \Lambda$, by Lemma 3.4 it suffices to separate $S \cap G_{\mathcal{O}_{k}}$ in $G_{\mathcal{O}_{k}} \cap \Lambda$. Since $S \cap G_{\mathcal{O}_{k}}$ is a subgroup of $B_{\mathcal{O}_{k}}$, by Theorem 3.1, $S \cap G_{\mathcal{O}_{k}}$ is separable in $G_{\mathcal{O}_{k}}$. Thus, $S \cap G_{\mathcal{O}_{k}}$ is separable in $G_{\mathcal{O}_{k}} \cap \Lambda$.

As a result of Corollary 3.10 every corollary and theorem stated below implies the same result for any $k$-arithmetic subgroup in $G$. Consequently, we only state the results for group of $k$-integral points. The connected assumption is unnecessary since every $k$-algebraic group has finitely many connected components (see [26, p. 51]).

One corollary to Theorem 3.1 is:

Corollary 3.11 Let $G$ be a connected $k$-algebraic group.

(a) If $U<G$ is a maximal unipotent subgroup, then every subgroup of $U_{\mathcal{O}_{k}}$ is separable in $G_{\mathcal{O}_{k}}$.

(b) If $T<G$ is a maximal torus, then every subgroup of $T_{\mathcal{O}_{k}}$ is separable in $G_{\mathcal{O}_{k}}$.

(c) If $S<G_{\mathcal{O}_{k}}$ is a solvable subgroup, then $S$ is separable in $G_{\mathcal{O}_{k}}$.

Proof (a) and (b) follow immediately from Theorem 3.1 since $U$ and $T$ are contained in a Borel subgroup. For (c), since every solvable subgroup is virtually contained in a Borel subgroup (see [4, p. 137]), by Lemma 3.2, it suffices to separate $S \cap B$ in $G_{\mathcal{O}_{k}}$. The latter is done using Theorem 3.1 .

For a $k$-algebraic group $G$, by an arithmetic $G$-orbifold, we mean a topological manifold of the form $G / \Lambda$, where $\Lambda$ is an arithmetic lattice in $G$. 
Theorem 3.12 Let $\rho: N \longrightarrow M$ be a $\pi_{1}$-injective immersion of an infrasolv manifold $N$ into an arithmetic $G$-orbifold $M$. Then there exists a finite cover $\psi: M^{\prime} \longrightarrow M$ such that $\rho$ lifts to an embedding.

Proof The map $\rho$ induces a homomorphism $\rho_{*}: \pi_{1}(N) \longrightarrow \pi_{1}(M)$. Since $N$ is an infrasolv manifold, $\rho_{*}\left(\pi_{1}(N)\right)$ is a solvable subgroup of $\pi_{1}(M)$. Since $\pi_{1}(M)=\Lambda$, for some arithmetic lattice in $G$, by Corollary [3.11] $\rho_{*}\left(\pi_{1}(M)\right)$ is separable in $\pi_{1}(M)$. It now follows by a standard argument (see [18]) that $\rho$ can be promoted to an embedding in some finite covering of $M$.

\section{The stabilizer subgroup separability theorem}

In this section we prove Theorem 1.3 and corollaries specific to lattices in the isometry group of hyperbolic space.

\subsection{Stabilizer subgroup separability}

As mentioned in $\S 2.7$, there is a simple trichotomy for the stabilizer groups of light-like vectors for $X$-hyperbolic lattices. For a lattice $\Lambda<\operatorname{Isom}\left(\mathbf{H}_{X}^{n}\right)$ and $v \in \partial \mathbf{H}_{X}^{n}$, exactly one of the following holds:

(1) $\triangle_{v}(\Lambda)$ is finite.

(2) $\triangle_{v}(\Lambda)$ is virtually cyclic with maximal cyclic subgroup generated by a loxodromic isometry.

(3) $\triangle_{v}(\Lambda)$ is an AC-group modelled on the $X$-Heisenberg group $\mathfrak{N}_{\ell n-1}(X)$.

Proof of Theorem 1.3 To prove Theorem [1.3, we split our consideration naturally into three cases depending on the above trichotomy.

Since $X$-hyperbolic lattices are residually finite it follows easily from Lemma 3.2 that subgroups in case (1) are separable. For $X=\mathbf{R}$ or $\mathbf{C}$, case (2) follows exactly the proof in [14] on noting $\mathrm{GL}(n ; \mathbf{C}) \longrightarrow \mathrm{GL}(2 n ; \mathbf{R})$. For $X=\mathbf{H}$ or $\mathbf{O}$, since every lattice in $\operatorname{Isom}\left(\mathbf{H}_{\mathbf{H}}^{n}\right)$ and $\operatorname{Isom}\left(\mathbf{H}_{\mathbf{O}}^{2}\right)$ is arithmetic, we can apply Corollary 3.11 (c) to separate.

For (3), as peripheral subgroups are virtually unipotent, Corollary 3.11 handles this case. To be complete, we first realize the arithmetic lattice $\Lambda$ as a subgroup of $\operatorname{GL}(m ; \mathbb{Q})$ with a finite index subgroup in $\operatorname{GL}(m ; \mathbb{Z})$ and finish by applying Corollary 3.10 with Corollary 3.11 . 
Remark In 14, Hamilton proved that in a cocompact lattice $\Lambda<\operatorname{Isom}\left(\mathbf{H}_{\mathbf{R}}^{n}\right)$, every virtually abelian subgroup is separable. As her proof does not require arithmeticity, our proof of Theorem 1.3 uses arithmeticity only in (3).

Corollary 4.1 Let $\Lambda$ be an arithmetic real hyperbolic lattice and $A$ an abelian subgroup. Then $A$ is separable in $\Lambda$.

The analog of abelian subgroups in the complex, quaternionic, octonionic settings are nilpotent subgroups. In the complex setting, we have:

Corollary 4.2 Let $\Lambda$ be an arithmetic complex hyperbolic lattice and $N$ a nilpotent subgroup. Then $N$ is separable in $\Lambda$.

Since all lattices in $\operatorname{Isom}\left(\mathbf{H}_{\mathbf{H}}^{n}\right)$ and $\operatorname{Isom}\left(\mathbf{H}_{\mathbf{O}}^{2}\right)$ are arithmetic, we may drop the arithmeticity condition to obtain:

Corollary 4.3 Let $\Lambda$ be a lattice in $\operatorname{Isom}\left(\mathbf{H}_{\mathbf{H}}^{n}\right)$ or $\operatorname{Isom}\left(\mathbf{H}_{\mathbf{O}}^{2}\right)$ and $N$ a nilpotent subgroup. Then $N$ is separable in $\Lambda$.

\section{$5 \quad$ A necessary and sufficient condition for arithmetic admissibility}

The goal of this section is to give a classification of cusp cross-sections of arithmetic $X$-hyperbolic $n$-orbifolds. By Theorem 1.5, we are reduced to classifying AB-groups which admit injections into arithmetic $X$-hyperbolic lattices. The main point of this section is to prove that this is equivalent to constructing injections into arithmetically defined subgroups of unitary affine groups. The latter groups are easier to work with in regard to this problem, as the generalized Bieberbach theorems ensure the existence of injections. The proof of this reduction relies on being able to realize unitary affine groups as algebraic subgroups in the isometry group of $X$-hyperbolic space. In total, this section is straightforward with the bulk of the material consisting of terminology, notation, and formal manipulation. We hope the main point of this section is not lost in this. 


\subsection{Characterization of noncocompact arithmetic lattices}

In this subsection, we give the classification of noncocompact arithmetic $X-$ hyperbolic $n$-lattices. This is originally due to Weil [35]. We refer the reader to [24] for a proof.

Theorem 5.1 Let $\Lambda$ be a noncocompact arithmetic lattice in $\operatorname{Isom}\left(\mathbf{H}_{X}^{n}\right)$.

(a) If $X=\mathbf{R}$, then $\Lambda$ is conjugate to an arithmetic lattice in $\mathrm{O}(B)$, where $B$ is a signature $(n, 1)$ bilinear form defined over $\mathbb{Q}$.

(b) If $X=\mathbf{C}$, then $\Lambda$ is conjugate to an arithmetic lattice in $\mathrm{U}(H)$, where $H$ is a Hermitian form of signature $(n, 1)$ defined over an imaginary quadratic number field.

(c) If $X=\mathbf{H}$, then $\Lambda$ is conjugate to an arithmetic lattice in $\mathrm{U}(H)$, where $H$ is a Hermitian form of signature $(n, 1)$ defined over a definite quaternion algebra with Hilbert symbol $\left(\frac{-a,-b}{\mathbb{Q}}\right)$ for $a, b \in \mathbb{N}$.

\subsection{Algebraic structure of unitary affine groups}

Recall for each maximal compact subgroup $M(X)$ of Aut $\left(\mathfrak{N}_{\ell n-1}\right)$, we defined the unitary affine group $U_{M}(n-1 ; X)$ to be $\mathfrak{N}_{\ell n-1}(X) \rtimes M(X)$. The algebraic structure of these groups is completely determined by the algebraic structure of the maximal compact subgroup. Specifically, $U_{M}(n-1 ; X)$ is $k$-algebraic if and only if $M$ is $k$-algebraic. In turn, the algebraic structure of $M$ is controlled by the finite index subgroup $\mathrm{U}\left(H_{M}\right)$. For these groups, $\mathrm{U}\left(H_{M}\right)$ is $k$-algebraic if and only if $H_{M}$ is defined over $k$.

In the real setting, these groups are of the form $\mathrm{O}\left(B_{\infty}\right)$, where $B_{\infty}$ is a symmetric, positive definite bilinear form and the form $B_{\infty}$ will be defined over a subfield $k \subset \mathbf{R}$. In the complex setting, these groups are of the form $\mathrm{U}\left(H_{\infty}\right)$, where $H_{\infty}$ is a Hermitian form of signature $(n-1,0)$ and $H_{\infty}$ will be defined over a subfield $k \subset \mathbf{C}$. In the quaternionic setting, these groups are of the form $\mathrm{U}\left(H_{\infty}\right)$, where $H_{\infty}$ is a Hermitian form of signature $(n-1,0)$ and $H_{\infty}$ will be defined over a subalgebra $\mathcal{A} \subset \mathbf{H}$. Our only interest is when $k$ is a number

field in the first two settings or $\mathcal{A}$ is a quaternion algebra defined over a number field in the last setting. 


\subsection{Arithmetically defined subgroups of unitary affine groups}

For an AB-group $\Gamma$ modelled on $\mathfrak{N}_{\ell n-1}(X)$, we saw in $\S 2.6$ that $\Gamma$ can be conjugated into a subgroup of a unitary affine group $U_{M}(n-1 ; X)$ for any $M(X)$. If this unitary affine group is $k$-algebraic and $\Gamma$ is contained in the $k$-points, we say that $\Gamma$ is $k$-defined. When $\Gamma$ is commensurable with the $\mathcal{O}_{k}$-points $\left(\mathcal{O}_{k}\right.$ is either the ring of integers of $k$ or a maximal order in the quaternion algebra), we say that $\Gamma$ is a $k$-arithmetic subgroup. Note that if $\Gamma$ is $k$-defined, then by conjugating by a Heisenberg dilation, we can arrange for $\Gamma$ to be commensurable with a subgroup of the $\mathcal{O}_{k}$-points of the unitary affine group.

\subsection{The quaternionic setting}

In the quaternionic setting, we can realize $U_{M}(n-1 ; \mathbf{H})$ as $\widehat{k}$-algebraic subgroup of $\operatorname{GL}(m ; \mathbf{R})$, where $\widehat{k}$ is the field for which the quaternion algebra $\mathcal{A}$ is defined. For a maximal order $\mathcal{O}$ in $\mathcal{A}$ (see [21]), if $\Gamma$ has a finite index in the $\mathcal{O}$-points of some unitary affine group $U_{M}(n-1 ; \mathbf{H})$, when we realize $U_{M}(n-1 ; \mathbf{H})$ as

a $\widehat{k}$-algebraic group, $\Gamma$ will have a finite index subgroup in the $\mathcal{O}_{\widehat{k}}$-points of this group.

In our notation, we will refer to $U_{M}(n-1 ; \mathbf{H})$ as being $\mathcal{A}$-defined, subgroups $\Gamma$ which are commensurable with $\mathrm{U}(n-1 ; \mathcal{O})$ for some maximal order $\mathcal{O}$ as being $\mathcal{A}$-arithmetic, and homomorphisms as being $\mathcal{A}$-defined. Since when we realize $\mathrm{U}(n-1 ; \mathbf{H})$ as a $\widehat{k}$-algebraic group, these definitions correspond to the standard algebraic definitions (over the field $\widehat{k}$ ), this is only a slight abuse of notation.

\section{$5.5 k$-monomorphisms of unitary affine groups into the isome- try group}

In this subsection, we characterize when a unitary affine group admits a $k-$ algebraic structure via embeddings into the isometry group of $X$-hyperbolic space.

Let $U_{M}(n-1 ; X)$ be a $k$-algebraic unitary affine group. Then $H_{M(X)}$, the associated Hermitian form for $M(X)$, is defined over $k$. Set $H=H_{M(X)} \oplus D_{2}$, with $H$ defined on $X^{n-1} \oplus X^{2}$ and $\left(X^{2}, D_{2}\right)$ is a $k$-defined $X$-hyperbolic plane. Finally, let $V_{\infty}$ denote the $H$-orthogonal complement in $X^{n+1}$ of a pair of $X$-linearly independent, $k$-defined, $H$-null vectors $v$ and $v_{0}$ in $\left(X^{2}, D_{2}\right)$. 
Let $\psi:\left(X^{n-1}, H_{M(X)}\right) \longrightarrow\left(V_{\infty}, H_{\mid V_{\infty}}\right)$ be any isometric isomorphism defined over $k$. This $X$-linear map induces a $k$-isomorphism

$$
\rho: U_{M}(n-1 ; X) \longrightarrow M N,
$$

where $N$ and $M$ are factors in the Iwasawa decomposition induced on $\operatorname{Stab}(v)$ with respect to the above pair of $H$-null vectors. Since both vectors are $k-$ defined, it follows that $M N$ is $k$-algebraic.

As a result of this discussion, we have the following proposition.

Proposition 5.2 $U_{M}(n-1 ; X)$ is a $k$-algebraic group if and only if there exists a Hermitian form $H$ of signature $(n, 1)$ defined over $k$ and a $k$-isomorphism $\rho: U_{M}(n-1 ; X) \longrightarrow M N<\operatorname{Isom}\left(\mathbf{H}_{X}^{n}\right)$ where $\mathbf{H}_{X}^{n}$ is modelled on $H$.

\subsection{A necessary and sufficient condition for arithmeticity}

In this subsection, we classify cusp cross-sections of arithmetic hyperbolic lattices. In the previous subsection, we related the algebraic structure of abstractly defined unitary affine groups via embeddings into the isometry group of $X$-hyperbolic space. In this subsection, we do the same for AB-groups.

We start with the following proposition which determines when an AB-group is $k$-defined.

Proposition 5.3 $\Gamma$ is a $k$-defined $A B$-group modelled on $\mathfrak{N}_{\ell n-1}(X)$ if and only if there exists a $k$-defined Hermitian form $H$ modelling $X$-hyperbolic $n$-space, a subgroup $\Lambda<\mathrm{U}(H ; k)$ commensurable with $\mathrm{U}\left(H ; \mathcal{O}_{k}\right)$, and an injection $\rho: \Gamma \longrightarrow \operatorname{Stab}(v) \cap \Lambda$ for some $k$-defined light-like vector $v$.

Proof For the direct implication, assume $\Gamma<U_{M}(n-1 ; X)$, for a $k$-defined unitary affine group. Let $\rho: U_{M}(n-1 ; X) \longrightarrow M N<\mathrm{U}(H)$ be a $k$-isomorphism given by Proposition [5.2. This gives us a $k$-monomorphism $\rho: U_{M}(n-1 ; X) \longrightarrow \mathrm{U}(H)$ of $k$-algebraic groups. By Lemma 2.2, there exists $\Lambda<\mathrm{U}(H ; k)$, commensurable with $\mathrm{U}\left(H ; \mathcal{O}_{k}\right)$ such that $\rho(\Gamma)<\Lambda$, as asserted.

For the reverse implication, we assume the existence of $H, \Lambda, \rho$, and $v$. Note that for the Fitting subgroup $L$ of $\Gamma, \rho(L)<N$, for some nilpotent factor of an Iwasawa decomposition. Since $L$ is Zariski dense in $N$ and consists of $k$-points, $N$ is a $k$-algebraic subgroup. Since $\rho(\Gamma)$ is virtually contained in $N$, $\rho(\Gamma)<M N$, for the compact factor $M$ of an Iwasawa decomposition $M A N$ of $\operatorname{Stab}(v)$. Since the group $M$ can be selected to be $k$-algebraic, we have $\rho(\Gamma)<M N$, where $M N$ is a $k$-algebraic unitary affine group, as desired. 
For an AB-group $\Gamma$ modelled on $\mathfrak{N}_{\ell n-1}(X)$, we say that $\Gamma$ is arithmetically admissible if there exists an arithmetic $X$-hyperbolic $n$-lattice $\Lambda$ such that $\Gamma$ is isomorphic to $\triangle_{v}(\Lambda)$. Altogether we have the following theorem which classifies the arithmetically admissible AB-groups (part (a) is proved in [20]).

Theorem 5.4 (Cusp classification theorem) Let $\Gamma$ be an AB-group modelled on $\mathfrak{N}_{\ell n-1}(X)$.

(a) For $X=\mathbf{R}, \Gamma$ is arithmetically admissible if and only if $\Gamma$ is a $\mathbb{Q}$ arithmetic subgroup in $\mathbf{R}^{n-1} \rtimes \mathrm{O}\left(B_{\infty}\right)$, where $B_{\infty}$ is a $\mathbb{Q}$-defined, positive definite, symmetric bilinear form on $\mathbf{R}^{n-1}$.

(b) For $X=\mathbf{C}, \Gamma$ is arithmetically admissible if and only if $\Gamma$ is a $k-$ arithmetic subgroup in a unitary affine group for some imaginary quadratic number field $k$.

(c) For $X=\mathbf{H}, \Gamma$ is arithmetically admissible if and only if $\Gamma$ is a $\mathcal{A}-$ arithmetic subgroup in a unitary affine group, for some quaternion algebra $\mathcal{A}$ with Hilbert symbol $\left(\frac{-a,-b}{\mathbb{Q}}\right)$ for $a, b \in \mathbb{N}$.

Proof The direct implication is immediate in all three case. For the converse, assume that $\Gamma$ is a $k$-arithmetic subgroup in a unitary affine group, where $k$ is as above. By Proposition [5.3 there exists a $k$-defined Hermitian form $H$ modelling $X$-hyperbolic $n$-space, a subgroup $\Lambda<\mathrm{U}(H ; k)$ commensurable with $\mathrm{U}\left(H ; \mathcal{O}_{k}\right)$, and an injection $\rho: \Gamma \longrightarrow \operatorname{Stab}(v) \cap \Lambda$ for some $k$-defined light-like vector $v . \quad \rho(\Gamma)$ must be a finite index subgroup of $\triangle_{v}(\Lambda)$ and by Theorem 5.1 $\Lambda$ is an arithmetic subgroup. In this injection we cannot ensure that $\rho(\Gamma)=\triangle_{v}(\Lambda)$. As $\Lambda$ is an arithmetic subgroup in the $k$-algebraic group $\mathrm{U}(H)$, by Theorem 1.3, we can find a finite index subgroup $\Pi<\Lambda$ such that $\rho(\Gamma)=\triangle_{v}(\Pi)$. Specifically, select a complete set of coset representatives for $\triangle_{v}(\Lambda) / \rho(\Gamma)$, say $\alpha_{1}, \ldots, \alpha_{r}$. By Theorem 1.3. there exists a finite index subgroup $\Pi$ of $\Lambda$ such that $\rho(\Gamma)<\Pi$ and for each $j=1, \ldots, r, \alpha_{j} \notin \Pi$. It then follows that $\triangle_{v}(\Pi)=\rho(\Gamma)$, as desired.

Remark Using the Bieberbach theorems, we can easily see from (a) that every Bieberbach group is arithmetically admissible. Altogether, this yields a slightly simpler proof of the main result in [20].

For an AB-group $\Gamma$ modelled on $\mathfrak{N}_{2 n-1}$, we say that the holonomy group $\theta$ of $\Gamma$ is complex if $\theta \subset \mathrm{U}\left(H_{M(X)}\right)<M(X)$ for the holonomy representation. Otherwise, we say that $\theta$ is anticomplex. We have the following alternative characterization based on the structure of the holonomy representation. 
Corollary 5.5 (Holonomy theorem) Let $\Gamma$ an AB-group modelled on $\mathfrak{N}_{2 n-1}$ with complex holonomy. Then $\Gamma$ is arithmetically admissible if and only if the holonomy representation $\varphi$ is conjugate to a representation

$$
\rho: \theta \longrightarrow \mathrm{GL}(n-1 ; k)
$$

for some imaginary quadratic number field.

Proof If $\Gamma$ is arithmetically admissible, then from Theorem 5.4 there exists a $k$-defined unitary affine group $U_{M}(n-1 ; k)$ such that $\Gamma$ is conjugate into $U_{M}(n-1 ; k)$ and commensurable with $U_{M}\left(n-1 ; \mathcal{O}_{k}\right)$, for some imaginary quadratic number field $k$. This yields an injective homomorphism

$$
\rho: \theta \longrightarrow M(k) .
$$

Since $\theta$ complex, $\rho(\theta)<\mathrm{U}\left(H_{M(X)} ; k\right)$, which is a subgroup of $\mathrm{GL}(n-1 ; k)$, as desired.

For the converse, assume that the holonomy representation of $\theta$ maps into $\mathrm{GL}(n-1 ; k)$, for some imaginary quadratic number field $k$. By taking the $\theta$-average of any $k$-defined Hermitian form, we see that this representation is contained in a $k$-defined unitary group $\mathrm{U}\left(H_{M(X)} ; k\right)$. Using this representation and a presentation for $\Gamma$, we get a system of linear homogenous equations with coefficients in $k$. Since $\rho$ is conjugate to the holonomy representation, by the generalized Bieberbach theorems, this system has a solution which yields a faithful representation into $\mathfrak{N}_{2 n-1}(k) \rtimes \mathrm{U}\left(H_{M(X)} ; k\right)$. By conjugating by a Heisenberg dilation to ensure that the Fitting subgroup consists of $k$-integral entries, we see that $\Gamma$ is $k$-arithmetic. Therefore, by Theorem 5.4, $\Gamma$ is arithmetically admissible.

\section{$6 \quad$ Families of examples}

In this section, we give examples which show that the characterization of arithmetic admissibility is nontrivial. These examples constitute a proof of Theorem 1.2

\subsection{Prime order holonomy}

Let $\Gamma$ be an AB-group modelled on $\mathfrak{N}_{2 n-1}$ with cyclic order $p$ holonomy, $C_{p}$, where $p$ is an odd prime. Note that this holonomy is necessarily complex and 
so acts trivially on the center of the Fitting subgroup. By taking the quotient of $\Gamma$ by its center, we get an $(2 n-2)$-dimensional Bieberbach group with $C_{p}$ holonomy. By the Bieberbach theorems, there exists a faithful representation of $C_{p}$ into $\mathrm{GL}(2 n-2 ; \mathbb{Z})$. This can occur only when $p-1 \leq 2 n-2$. That such AB-groups exist in dimension $2 n-1$ can be shown by explicit construction.

The following proposition shows that there are infinitely many AB-groups modelled on $\mathfrak{N}_{2 n-1}$ for infinitely many $n$ which are not arithmetic admissible.

Proposition 6.1 Let $\Gamma_{p}$ denote an $A B$-groups modelled on $\mathfrak{N}_{2 n-1}$ with holonomy $C_{p}$ and $2(n-1)=p-1$. If $\Gamma_{p}$ is arithmetically admissible, then $p \equiv 3$ $\bmod 4$.

Proof If $\Gamma_{p}$ is arithmetically admissible, by Corollary [5.5, there exists a faithful representation ( $k$ is an imaginary quadratic number field)

$$
\rho: C_{p} \longrightarrow \mathrm{GL}\left(\frac{p-1}{2} ; k\right) \text {. }
$$

Let $k_{\rho}$ denote the field generated by the traces of $\rho(\xi)$ for a generator $\xi \in C_{p}$ and note that $k_{\rho} \subset k$. The representation $\rho$ is conjugate to one which decomposes into a direct sum of characters $\chi_{j}: C_{p} \longrightarrow \mathbf{C}^{\times}$(see [8] or [30]). Each of these characters $\chi_{j}$ is of the form $\chi_{j}(\xi)=\zeta_{p}^{n_{j}}$. Therefore

$$
\operatorname{Tr}(\rho(\xi))=\sum_{j=1}^{\frac{p-1}{2}} \zeta_{p}^{n_{j}} .
$$

Since $\rho$ is faithful, for some $j, n_{j} \neq 0 \bmod p$. By considering the cyclotomic polynomial $\Phi_{p}(x)$, we deduce that $\operatorname{Tr}(\rho(\xi)) \notin \mathbb{Q}$ and so $k_{\rho}$ is a nontrivial extension of $\mathbb{Q}$. On the other hand, from the decomposition above, $k_{\rho} \subset \mathbb{Q}\left(\zeta_{p}\right)$. Since $[k: \mathbb{Q}]=2$, it must be that $k=k_{\rho}$. Hence $\mathbb{Q}\left(\zeta_{p}\right)$ contains an imaginary quadratic extension of $\mathbb{Q}$. By quadratic reciprocity, this can happen if and only if $p \equiv 3 \bmod 4$.

It is worth noting for $p \equiv 1 \bmod 4$, Corollary 5.5 can be used to show that such AB-groups are arithmetically admissible and the field $k$ is the unique imaginary quadratic number field in $\mathbb{Q}\left(\zeta_{p}\right)$. Moreover, the holonomy generator acts by the matrix $\operatorname{Res}_{\mathbb{Q}\left(\zeta_{p}\right) / k}\left(\zeta_{p}\right)$, where Res denotes the restriction of scalar operation.

Remark Note when $p>5$, we get an obstruction without appealing to Theorem [5.4. In this case, we have an injection

$$
\rho: C_{p} \longrightarrow \mathrm{U}(H ; k)<\mathrm{GL}\left(\frac{p+1}{2} ; k\right) \text {. }
$$


So long as $(p+1) / 2<p-1$, this implies $p \equiv 3 \bmod 4$.

In the quaternionic setting, take $\Gamma$ modelled on $\mathfrak{N}_{7}(\mathbf{H})$ with $C_{5}$-holonomy where the action of $C_{5}$ on the center of $\mathfrak{N}_{7}(\mathbf{H})$ is trivial. If $\Gamma$ is arithmetically admissible, we obtain an injection of $C_{5}$ into $\mathcal{A}$, a definite quaternion algebra over $\mathbb{Q}$. However, this is impossible since such $\mathcal{A}$ do not contain elements of order five (see [33]). Thus $\Gamma$ cannot be arithmetically admissible. This yields:

Proposition 6.2 There exists infinitely many nonarithmetically admissible AB-groups modelled on $\mathfrak{N}_{7}(\mathbf{H})$.

In the next subsection, by taking products of this example with quaternionic nil-tori, we can construct infinitely many examples in $\mathfrak{N}_{4 n+3}(\mathbf{H})$ for $4 n+3>7$.

\subsection{Central products}

In the complex setting, we get arithmetically inadmissible examples in every dimension greater than two by constructing new AB-groups from a pair of ABgroups. The details are as follows.

Let $\Gamma_{1}$ and $\Gamma_{2}$ denote a pair of AB-groups modelled on $\mathfrak{N}_{2 n_{1}-1}$ and $\mathfrak{N}_{2 n_{2}-1}$, respectively. We define $\Gamma=\Gamma_{1} \times{ }_{c} \Gamma_{2}$ to be the group $\Gamma_{1} \times \Gamma_{2} / N_{\Gamma_{1} \times \Gamma_{2}}\left(\left\langle c_{1} c_{2}^{-1}\right\rangle\right)$, where $c_{j}$ is the generator of the center of the Fitting subgroup $L_{j}$ of $\Gamma_{j}$ and $N_{\Gamma_{1} \times \Gamma_{2}}\left(c_{1} c_{2}^{-1}\right)$ denotes the normal closure of $\left\langle c_{1} c_{2}^{-1}\right\rangle$ in $\Gamma_{1} \times \Gamma_{2}$. We call this the central product of $\Gamma_{1}$ and $\Gamma_{2}$.

Lemma 6.3 If $\theta_{1}$ and $\theta_{2}$, the respective holonomy groups, are either both complex or both anticomplex, then $\Gamma_{1} \times_{c} \Gamma_{2}$ is an AB-group modelled on $\mathfrak{N}_{2\left(n_{1}+n_{2}\right)-1}$.

Proof As we only make use of the case when the holonomy groups are complex, we leave the anticomplex case for the reader. To begin, we have natural inclusions of $\mathfrak{N}_{2 n_{1}-1}$ and $\mathfrak{N}_{2 n_{2}-1}$ into $\mathfrak{N}_{2\left(n_{1}+n_{2}\right)-1}$ induced by

$$
\rho_{j}: \mathbf{C}^{n_{j}} \longrightarrow \mathbf{C}^{n_{1}} \oplus \mathbf{C}^{n_{2}} \text {. }
$$

In fact, this yields inclusions of $\mathbf{C}^{n_{j}} \rtimes \mathrm{GL}\left(n_{j} ; \mathbf{C}\right)$ into $\mathbf{C}^{n_{1}+n_{2}} \rtimes \mathrm{GL}\left(n_{1}+n_{2} ; \mathbf{C}\right)$. As a result, we have an injective homomorphism of $\Gamma_{1} \times_{c} \Gamma_{2}$ onto an AB-group in $\operatorname{Aff}\left(\mathfrak{N}_{2\left(n_{1}+n_{2}\right)-1}\right)$. By carefully selecting the maps $\rho_{j}$, we can ensure that the induced maps agrees on the center of the Fitting subgroups of $\Gamma_{1}$ and $\Gamma_{2}$. With this selection, the induced map becomes an isomorphism of $\Gamma_{1} \times{ }_{c} \Gamma_{2}$ with an AB-group in $\mathfrak{N}_{2\left(n_{1}+n_{2}\right)-1}$, as desired. 
Remark In the complex holonomy case, the group $\left\langle c_{1} c_{2}^{-1}\right\rangle$ is normal.

Using central products we can construct many arithmetically inadmissible ABgroups. We summarize this in the following theorem which proves Theorem 1.2 (a).

Theorem 6.4 (Central product theorem) Let $\Gamma_{1}$ and $\Gamma_{2}$ be AB-groups modelled on $\mathfrak{N}_{2 n_{1}-1}$ and $\mathfrak{N}_{2 n_{2}-1}$, defined over $k_{1}$ and $k_{2}$, respectively. Assume $\Gamma_{1}$ and $\Gamma_{2}$ are both complex or anticomplex.

(a) $\Gamma_{1} \times_{c} \Gamma_{2}$ is $k_{1} k_{2}$-defined.

(b) $\Gamma_{1} \times{ }_{c} \Gamma_{2}$ is $k$-arithmetic if and only if both $\Gamma_{1}$ and $\Gamma_{2}$ are $k$-arithmetic.

(c) There exist arithmetically inadmissible AB-groups modelled on $\mathfrak{N}_{2 n-1}$ for all $n \geq 3$.

Proof For (a), let $M_{1}(X)$ and $M_{2}(X)$ denote the maximal compact groups defined over $k_{1}$ and $k_{2}$ and for which $\Gamma_{j}$ injects into the $k_{j}$-points of $\mathfrak{N}_{2 n_{j}-1}(X) \rtimes M_{j}(X)$. Associated to each of these maximal compact groups is a $k_{j}$-defined Hermitian form $H_{j}$. Let $H=H_{1} \oplus H_{2}$ and $M(X)$ be the associated maximal compact subgroup. $M(X)$ is $k_{1} k_{2}$-define and we have an injection (into the $k_{1} k_{2}$-points) $\rho: \Gamma_{1} \times_{c} \Gamma_{2} \longrightarrow \mathfrak{N}_{2\left(n_{1}+n_{2}\right)-1}(X) \rtimes M(X)$. Thus $\Gamma_{1} \times_{c} \Gamma_{2}$ is $k_{1} k_{2}$-defined.

For (b), if $\Gamma=\Gamma_{1} \times_{c} \Gamma_{2}$ is $k$-arithmetic, then $\Gamma$ is isomorphic to a maximal peripheral subgroup of an arithmetic lattice $\Lambda$ in $\mathrm{U}(H)$, where $H$ is a signature $\left(n_{1}+n_{2}, 1\right)$ Hermitian form defined over an imaginary quadratic number field $k$. It must be that $\Gamma_{j}$ injects into a subgroup $\Lambda_{j}$ of $\Lambda$ which is maximal with respect to stabilizing a $k$-defined complex subspace $\mathbf{C}^{n_{j}, 1}$. As $\Lambda_{j}$ is an arithmetic lattice in a smaller isometry group (whose model form is the restriction of $H$ to the complex subspace $\mathbf{C}^{n_{j}, 1}$ ), this shows that $\Gamma_{j}$ is $k$ arithmetic for $j=1,2$. The reverse implication follows immediately from (a).

For (c), by Proposition 6.1 there exists an arithmetically inadmissible ABgroup modelled on $\mathfrak{N}_{5}$. To obtain examples in higher dimensions, we take central products of this example with other AB-groups and apply (b).

The quaternionic setting can be handled similarly. We construct examples in every dimension by taking central products with the inadmissible example $\Gamma$ in $\mathfrak{N}_{7}(\mathbf{H})$ given above.

Corollary 6.5 There exist arithmetically inadmissible AB-groups modelled on $\mathfrak{N}_{4 n-1}(\mathbf{H})$ for all $n \geq 2$. 
Remark Theorem 1.2 (b) shows that a quaternionic version of Conjecture 1.1 (a) is false. Namely, there exist almost flat manifolds modelled on the quaternionic Heisenberg group which cannot arise as the a cusp cross-section of a 1-cusped quaternionic hyperbolic manifold. The author has been informed by Walter Neumann and Alan Reid that nil 3-manifold groups exist which cannot arise as a cusp cross-section of a 1-cusped complex hyperbolic 2-manifold. In fact, Yoshinobu Kamishima [16] proves that no nil 3-manifold group with nontrivial holonomy can arise as a cusp cross-section of a 1-cusped complex hyperbolic 2-manifold.

\section{Analysis in low complex dimensions}

In this section, we work out the specific details of Theorem [5.4 for closed nil 3-manifold groups. In particular, we prove Corollary 1.6. Before we undertake this task, it is worth noting that the validity of Corollary [1.6 follows almost at once from Corollary [5.5. The only ingredient needed is the classification of nil 3-manifold groups given below. However, in this section we explicitly construction the desired $k$-integral representations for such groups to better illustrate the proof of Theorem 5.4.

\subsection{Some preliminary material}

In this subsection, we give an alternative (more traditional) description of nil 3-manifolds and list the classification of closed nil 3-manifold groups. See 29] for a reference on this material.

The 3-dimensional Heisenberg group also has a definition as

$$
\mathfrak{N}_{3}=\left\{\left(\begin{array}{lll}
1 & x & t \\
0 & 1 & y \\
0 & 0 & 1
\end{array}\right): x, y, t \in \mathbf{R}\right\} .
$$

We will identify $S^{1}$ with the rotations in the $x y$-plane. An orientable nil 3manifold is a manifold of the form $\mathfrak{N}_{3} / \Gamma$, where $\Gamma$ is a discrete subgroup of $\mathfrak{N}_{3} \rtimes S^{1}$ which acts freely. As we will have need for this in the sequel, we must also consider an orientation reversing involution given by

$$
\tau\left(\begin{array}{lll}
1 & 0 & 0 \\
0 & 1 & y \\
0 & 0 & 1
\end{array}\right)=\left(\begin{array}{ccc}
1 & 0 & 0 \\
0 & 1 & -y \\
0 & 0 & 1
\end{array}\right), \quad \tau\left(\begin{array}{ccc}
1 & x & 0 \\
0 & 1 & 0 \\
0 & 0 & 1
\end{array}\right)=\left(\begin{array}{lll}
1 & x & 0 \\
0 & 1 & 0 \\
0 & 0 & 1
\end{array}\right) .
$$


As automorphisms of lattices of $\mathfrak{N}_{3}$ uniquely determine automorphisms of $\mathfrak{N}_{3}$ by Mal'cev rigidity, this determines a continuous isomorphism of $\mathfrak{N}_{3}$. A nil 3-manifold is a manifold of the form $\mathfrak{N}_{3} / \Gamma$, where $\Gamma$ is a discrete subgroup of $\left\langle\mathfrak{N}_{3} \rtimes S^{1}, \widetilde{\iota}\right\rangle$ which acts freely.

Remark This terminology follows [29] and 31. However, in our terminology which follows 9], such manifolds should be called infranil 3-manifolds. We adhere to the first in this section and hope no confusion arises from this.

In $\S 2$, we gave a general definition of the Heisenberg group. That these two definitions coincide follows from the fact that both groups are connected, simply connected 2-step nilpotent Lie groups of dimension three and there is a unique such Lie group (up to Lie isomorphism) that has these properties. More generally, any simply connected, connected, 2-step nilpotent group with 1dimensional center is uniquely determined (see [24]) and is Lie isomorphic to the the Heisenberg group which we defined in $\S 2$.

In this form, we identify $S^{1}$ with $\mathrm{U}(1)$, where $\mathrm{U}(1)$ acts (as above) by

$$
U(z, t)=(U z, t) .
$$

We also identify $\iota$ and $\widetilde{\iota}$, where $\iota$ is the isometry induced by complex conjugation. When the nil 3 -manifold is closed, $\mathfrak{N}_{3} \cap \Gamma$ must be finite index in $\Gamma$ and $\mathfrak{N}_{3} /\left(\mathfrak{N}_{3} \cap \Gamma\right)$ must be compact.

The following is a complete list of closed nil 3-manifold groups (see [9, p. 159$166])$.

with $k \in \mathbb{N}$.

$$
\left\langle a, b, c:[b, a]=c^{k},[c, a]=[c, b]=1\right\rangle .
$$

(2)

$$
\begin{gathered}
\left\langle a, b, c, \alpha:[b, a]=c^{k},[c, a]=[c, b]=[\alpha, c]=1, \alpha a=a^{-1} \alpha\right. \\
\left.\alpha b=b^{-1} \alpha, \alpha^{2}=c\right\rangle,
\end{gathered}
$$

with $k \in 2 \mathbb{N}$.

(3)

$$
\begin{gathered}
\left\langle a, b, c, \alpha:[b, a]=c^{2 k},[c, a]=[c, b]=[a, \alpha]=1, \alpha c=c^{-1} \alpha\right. \\
\left.\alpha b=b^{-1} \alpha c^{-k}, \alpha^{2}=a\right\rangle
\end{gathered}
$$

with $k \in \mathbb{N}$. 
$(4)$

$$
\begin{aligned}
\langle a, b, c, \alpha, \beta: & {[b, a]=c^{2 k},[c, a]=[c, b]=[c, \alpha]=[a, \beta]=1, } \\
& \beta c=c^{-1} \beta, \alpha a=a^{-1} \alpha c^{k}, \alpha b=b^{-1} \alpha c^{-k} \\
& \left.\alpha^{2}=c, \beta^{2}=a, \beta b=b^{-1} \beta c^{-k}, \alpha \beta=a^{-1} b^{-1} \beta \alpha c^{-k-1}\right\rangle,
\end{aligned}
$$

with $k \in 2 \mathbb{N}$.

(5)

$$
\begin{gathered}
\left\langle a, b, c, \alpha:[b, a]=c^{k},[c, a]=[c, b]=[c, \alpha]=1, \alpha a=b \alpha,\right. \\
\left.\alpha b=a^{-1} \alpha, \alpha^{4}=c^{p}\right\rangle,
\end{gathered}
$$

with $k \in 2 \mathbb{N}$ and $p=1$ or $k \in 4 \mathbb{N}$ and $p=3$.

(6)

$$
\begin{gathered}
\left\langle a, b, c, \alpha:[b, a]=c^{k},[c, a]=[c, b]=[c, \alpha]=1, \alpha a=b \alpha c^{k_{1}},\right. \\
\left.\alpha b=a^{-1} b^{-1} \alpha, \alpha^{3}=c^{k_{2}}\right\rangle
\end{gathered}
$$

with $k>0$ and

$$
k \equiv 0 \quad \bmod 3, \quad k_{1}=0, k_{2}=1, \text { or } k \equiv 0 \quad \bmod 3, \quad k_{1}=0, k_{2}=2,
$$

or

$$
k \equiv 1,2 \quad \bmod 3, \quad k_{1}=1, k_{2}=1 .
$$

(7)

$$
\begin{gathered}
\left\langle a, b, c, \alpha:[b, a]=c^{k},[c, a]=[c, b]=[c, \alpha]=1, \alpha a=a b \alpha,\right. \\
\left.\alpha b=a^{-1} \alpha, \alpha^{6}=c^{k_{1}}\right\rangle,
\end{gathered}
$$

with $k>0$ and

$$
k \equiv 0 \quad \bmod 6, \quad k_{1}=1, \text { or } k \equiv 4 \bmod 6, \quad k_{1}=1,
$$

or

$$
k \equiv 0 \quad \bmod 6, \quad k_{1}=5, \text { or } k \equiv 2 \bmod 6, \quad k_{1}=5
$$

\subsection{Nil 3-manifolds}

Let $M$ be a closed nil 3-manifold with $\pi_{1}(M)=\Gamma$. By Theorem 2.1 in order to prove Corollary 1.6] it suffices to show that $\Gamma \cong \triangle(\Lambda)$, where $\Lambda$ is an arithmetic lattice in $\operatorname{Isom}\left(\mathbf{H}_{\mathbf{C}}^{2}\right)$. In fact, by Theorem 1.5 it suffices to construct an injective homomorphism $\varphi: \Gamma \longrightarrow \triangle(\Lambda)$.

Let $\mathfrak{N}(3)=\mathfrak{N}_{3} \rtimes \mathrm{U}(1)$ and $\iota$ be the isometry of $\mathbf{H}_{\mathbf{C}}^{2}$ induced by conjugation. For a subring $R \subset \mathbf{C}$, we define $\mathfrak{N}_{3}(R)=R \times \operatorname{Im} R$ with the induced group operation and set $\mathfrak{N}(3, R)=\mathfrak{N}_{3}(R) \rtimes \mathrm{U}(1 ; R)$.

For the statement, let $\zeta_{3}$ be a primitive third root of unity, say

$$
\zeta_{3}=-1 / 2+\sqrt{-3} / 2 .
$$


Theorem 7.1 Let $M$ be a closed nil 3-manifold $M$ and $\Gamma=\pi_{1}(M)$. Then there exists a faithful representation $\varphi: \Gamma \longrightarrow\left\langle\mathfrak{N}\left(3, \mathcal{O}_{k}\right), \iota\right\rangle$ with $k=\mathbb{Q}(i)$ or $\mathbb{Q}\left(\zeta_{3}\right)$.

Proof We begin by summarizing the strategy of the proof, which depends heavily on the list of presentations for the fundamental group of a closed nil 3manifold in previous subsection. The idea is to show that an injective homomorphism on the Fitting subgroup $\langle a, b, c\rangle$ can be promoted to the full 3-manifold group (see 9, Thm 3.1.3]). To get a representation with the coefficients in $\mathbb{Z}[i]$ or $\mathbb{Z}\left[\zeta_{3}\right]$, we are reduced to solving some simple equations. The details are as follows.

In the lemma below, let $p_{1}: \mathfrak{N}_{3} \longrightarrow \mathbf{C}$ be projection onto the first factor.

\section{Lemma 7.2}

(a) Let $a$ and $b$ be as above and $\rho:\langle a, b, c\rangle \longrightarrow \mathfrak{N}_{3}$ be a homomorphism. If $p_{1}(\rho(a))$ and $p_{1}(\rho(b))$ are $\mathbb{Z}$-linearly independent and $c \notin$ ker $\rho$, then $\rho$ is injective.

(b) Let $\rho: \Gamma \longrightarrow\langle\mathfrak{N}(3), \iota\rangle$ be a homomorphism. If $\rho^{-1}(\rho(\langle a, b, c\rangle))=\langle a, b, c\rangle$, and $\rho_{\mid\langle a, b, c\rangle}$ is an injective homomorphism, then $\rho$ is an injective homomorphism.

Proof For (a), let $w \in \operatorname{ker} \rho$ and write $w=a^{n_{1}} b^{n_{2}} c^{n_{3}}$. Also, let $\rho(a)=\left(v_{1}, t_{a}\right), \rho(b)=\left(v_{2}, t_{b}\right)$. Since $[a, b]=c^{k}$, it must be that $\rho(c)=(0, s)$ as $\left[\mathfrak{N}_{3}, \mathfrak{N}_{3}\right]=\{(0, t): t \in \mathbf{R}\}$. With this said, we see that

$$
\rho(w)=\left(n_{1} v_{1}+n_{2} v_{2}, n_{1} t_{a}+n_{2} t_{b}+2 \operatorname{Im}\left\langle n_{1} v_{1}, n_{2} v_{2}\right\rangle+n_{3} s\right) .
$$

Since $w \in \operatorname{ker} \rho, n_{1} v_{1}+n_{2} v_{2}=0$. The $\mathbb{Z}$-linear independence of $v_{1}$ and $v_{2}$ implies $n_{1}=n_{2}=0$. Therefore $n_{3} s=0$, and so $n_{3}=0$, as $s \neq 0$.

For (b), let $w \in \operatorname{ker} \rho$ and write

$$
w=a^{n_{1}} b^{n_{2}} c^{n_{3}} \alpha^{s_{1}} \beta^{s_{2}}, \quad n_{1}, n_{2}, n_{3}, s_{1}, s_{2} \in \mathbb{Z} .
$$

Since $\rho(w)=1$, using the above form for $w$, we have

$$
\rho\left(a^{n_{1}} b^{n_{2}} c^{n_{3}}\right)=\rho\left(\alpha^{-s_{1}} \beta^{-s_{2}}\right) .
$$

Since $\rho^{-1}(\rho(\langle a, b, c\rangle))=\langle a, b, c\rangle$, it must be that $\alpha^{s_{1}} \beta^{s_{2}} \in\langle a, b, c\rangle$. Thus $w \in\langle a, b, c\rangle$. Since $\rho_{\mid\langle a, b, c\rangle}$ is one-to-one, $w=1$, as desired. 
Remark Notice that for (b), we only require that if $\rho\left(\alpha^{s_{1}} \beta^{s_{2}}\right) \in \rho(\langle a, b, c\rangle)$ then $\alpha^{s_{1}} \beta^{s_{2}} \in\langle a, b, c\rangle$. Moreover, we only need to check for $s_{1} \in\left\{1, \ldots, k_{\alpha}\right\}$ and $s_{2} \in\left\{1, \ldots, k_{\beta}\right\}$ where $k_{\varepsilon}$ is the first integer such that $\varepsilon^{k_{\varepsilon}} \in\langle a, b, c\rangle, \varepsilon=\alpha$ or $\beta$.

Let $L=\langle a, b, c\rangle$ and define two homomorphisms $\varphi_{3}, \varphi_{4}: L \longrightarrow \mathfrak{N}_{3}$ by $\varphi_{j}(a)=(1,0)$ and $\varphi_{j}(b)=\left(\zeta_{j}, 0\right)$. This determines $c$, since some power of $c$ is a commutator of $a$ and $b$. By Lemma 7.2 (a), both maps are injective homomorphisms.

We extend this to $\Gamma$ by declaring

$$
\varphi_{j}(\alpha)=\left(z_{1}, t_{1}, \eta_{1}\right), \quad \varphi_{j}(\beta)=\left(z_{2}, t_{2}, \eta_{2}\right),
$$

where $z_{1}, z_{2} \in \mathbf{C}, t_{1}, t_{2} \in \mathbf{R}$, and $\eta_{1}, \eta_{2} \in\langle\mathrm{U}(1), \iota\rangle$.

To solve Equation (1), we simply use the presentations above to ensure that this yields a homomorphism. By applying Lemma 7.2 (b), one can see that these solutions yield injective homomorphisms. For clarity, we solve the equations for the second family (2) and give a list of the equations and solutions for the seventh family (7). We relegate the rest of the solutions to the appendix.

The second family has presentation

$$
\left\langle a, b, c, \alpha:[b, a]=c^{k},[c, a]=[c, b]=[c, \alpha]=1, \alpha a=a^{-1} \alpha, \alpha b=b^{-1} \alpha, \alpha^{2}=c\right\rangle
$$

with $k \in 2 \mathbb{N}$. For this family we take the map $\varphi_{4}$. First, consider the relation $[b, a]=c^{k}$. Then

$$
\begin{aligned}
{\left[\varphi_{4}(b), \varphi_{4}(a)\right] } & =[(i, 0,1),(1,0,1)] \\
& =(i+1,2 \operatorname{Im}\langle i, 1\rangle, 1)(-i-1,2 \operatorname{Im}\langle-i,-1\rangle, 1) \\
& =(0,4,1) .
\end{aligned}
$$

Since $\left[\varphi_{4}(b), \varphi_{4}(b)\right]=\varphi_{4}(c)^{k}$, it follows that $\varphi_{4}(c)=(0,4 / k, 1)$.

Next, consider the relation $\alpha^{2}=c$. We have

$$
\begin{aligned}
\left(z_{1}, t_{1}, \eta_{1}\right)\left(z_{1}, t_{1}, \eta_{1}\right) & =\left(z_{1}+\eta_{1} z_{1}, 2 t_{1}+2 \operatorname{Im}\left\langle z_{1}, \eta_{1} z_{1}\right\rangle, \eta_{1}^{2}\right) \\
& =(0,4 / k, 1) .
\end{aligned}
$$

In particular, $\eta_{1}^{2}=1$. If $\eta_{1}=1$, then the above injection would yield an isomorphism between a group in the first family with a group in the second family. This is impossible, therefore $\eta_{1}=-1$. By considering the first coordinate equation with $\eta_{1}=-1$, we get no information. The second coordinate equation is $2 t_{1}=4 / k$, therefore $t_{1}=2 / k$. One can now check that $[c, \alpha]=1$, regardless of $z_{1}$. 
Now, we take the relation $\alpha a=a^{-1} \alpha$. We have

$$
\left(z_{1}, 2 / k,-1\right)(1,0,1)=\left(z_{1}-1,2 / k-2 \operatorname{Im} z_{1},-1\right) .
$$

On the other hand,

$$
(-1,0,1)\left(z_{1}, 2 / k,-1\right)=\left(-1+z_{1}, 2 / k+2 \operatorname{Im} z_{1},-1\right) .
$$

The first and last coordinate equations yield no information, while the second coordinate equation yields $4 \operatorname{Im} z_{1}=0$. Hence $\operatorname{Im} z_{1}=0$.

Lastly, we have the relation $\alpha b=b^{-1} \alpha$. We have

$$
\left(z_{1}, 2 / k,-1\right)(i, 0,1)=\left(z_{1}-i, 2 / k+2 \operatorname{Re} z_{1},-1\right) .
$$

On the other hand,

$$
(-i, 0,1)\left(z_{1}, 2 / k,-1\right)=\left(-i+z_{1}, 2 / k-2 \operatorname{Re} z_{1},-1\right) .
$$

As above, the first and second coordinates yields no information, while the second coordinate implies that $\operatorname{Re} z_{1}=0$.

Hence, we deduce from the above computations, the desired homomorphism $\rho$ is defined by

$$
\begin{array}{lrl}
\rho(a)=(1,0,1), & \rho(b)=(i, 0,1) \\
\rho(c)=(0,4 / k, 1) & \rho(\alpha)=(0,2 / k,-1) .
\end{array}
$$

Notice that these solutions are in $\mathbb{Q}(i)$ and not $\mathbb{Z}[i]$. This is rectified by conjugating the above representation by a dilation of $2 k$. This dilation is linear on the first factor, quadratic on the second factor, and trivial on the third factor. The resulting faithful representation is

$$
\begin{aligned}
\rho(a) & =(2 k, 0,1), & \rho(b)=(2 k i, 0,1) \\
\rho(c) & =(0,16 k, 1) & \rho(\alpha)=(0,8 k,-1) .
\end{aligned}
$$

That this is faithful follows from Lemma 7.2 (b) once the conditions of this lemma have been verified. The injectivity of $\rho_{\mid\langle a, b, c\rangle}=\varphi_{4}$ follows from Lemma 7.2 (a). To check that $\rho\left(\rho^{-1}(\langle a, b, c\rangle)\right)=\langle a, b, c\rangle$, by the remark proceeding the proof of Lemma 7.2 it suffices to show that $\alpha \notin \rho\left(\rho^{-1}(\langle a, b, c\rangle)\right)$. The validity of this is obvious.

For the seventh family (7), we have the presentation

$$
\left\langle a, b, c, \alpha:[b, a]=c^{k},[c, a]=[c, b]=[c, \alpha]=1, \alpha a=a b \alpha, \alpha b=a^{-1} \alpha, \alpha^{6}=c^{k_{1}}\right\rangle
$$

with

$$
\begin{aligned}
& k \equiv 0 \quad \bmod 6, k_{1}=1, \text { or } k \equiv 4 \quad \bmod 6, k_{1}=1, \text { or } \\
& k \equiv 0 \quad \bmod 6, k_{1}=5, \text { or } k \equiv 2 \bmod 6, k_{1}=5 .
\end{aligned}
$$


We take $\varphi_{3}$ in this case. By considering all the relations, we get

$$
\begin{aligned}
(0,4 / k, 1) & =(0, s, 1) \\
\left(z_{1}+\eta_{1}, t_{1}+2 \operatorname{Im}\left\langle z, \eta_{1}\right\rangle, \eta_{1}\right) & =\left(1+\zeta_{3}+z_{1}, t_{1}+\right. \\
& \left.2 \operatorname{Im}\left\langle 1+\zeta_{3}, z_{1}\right\rangle+2 \operatorname{Im}\left\langle 1, \zeta_{3}\right\rangle+t_{1}, \eta_{1}\right) \\
\left(z_{1}+\eta_{1} \zeta_{3}, t_{1}+2 \operatorname{Im}\left\langle z_{1}, \eta_{1} \zeta_{3}\right\rangle, \eta_{1}\right) & =\left(-1+z_{1}, t_{1}+2 \operatorname{Im}\left\langle-1, z_{1}\right\rangle, \eta_{1}\right) .
\end{aligned}
$$

We omit the last relation $\alpha^{6}=c^{k_{1}}$ as it is quite long. Note the commutator relations (aside from $[b, a]=c^{k}$ ) are all trivially satisfied. Solving these equations and conjugating by a dilation of $12 k$ to get the coefficients in $\mathbb{Z}\left[\zeta_{3}\right]$, we have

$$
\begin{aligned}
\varphi_{3}(a) & =(12 k, 0,1), & \varphi_{3}(b)=\left(12 k \zeta_{3}, 0,1\right), \\
\varphi_{3}(c) & =(0,288 k \sqrt{3}, 1), & \varphi_{3}(\alpha)=\left(-6 k, 12 k\left(4 k_{1}+3 k\right) \sqrt{3}, \zeta_{6}\right) .
\end{aligned}
$$

That this is faithful follows from Lemma 7.2 and the remark following the proof. The other remaining families are handled similarly.

The proof is completed by following the injection $\rho$ from Theorem 7.1 with the injection

$$
\psi: \mathfrak{N}_{3} \rtimes\langle\mathrm{U}(1), \iota\rangle \longrightarrow \mathrm{U}(2,1)
$$

given by

$$
\left(\xi, t, \iota^{\varepsilon} U\right) \longmapsto\left(\begin{array}{ccc}
1 & \xi & \xi \\
-\bar{\xi} & 1-\frac{1}{2}\left(\|\xi\|^{2}-i t\right) & -\frac{1}{2}\left(\|\xi\|^{2}-i t\right) \\
\bar{\xi} & \frac{1}{2}\left(\|\xi\|^{2}-i t\right) & 1+\frac{1}{2}\left(\|\xi\|^{2}-i t\right)
\end{array}\right)\left(\begin{array}{ccc}
U & 0 & 0 \\
0 & 1 & 0 \\
0 & 0 & 1
\end{array}\right) \iota^{\varepsilon} .
$$

For completeness, we give all the linear representations in the appendix.

Remark Theorem 7.1 can be strengthened somewhat. Specifically, we can prove that there is a dense set of almost flat structures on any compact nil 3-manifold which can be realized in cusp cross-sections of arithmetic complex hyperbolic 2-orbifolds. We refer the reader for [24] for more on this.

\subsection{Nil 5-manifolds}

Using the list of isomorphism types of holonomy groups for nil 5-manifolds given in [10, we can carry out the same analysis.

Proposition 7.3 The only complex holonomy groups which yield arithmetically inadmissible groups are $C_{5}, C_{10}, C_{12}$ and $C_{24}$. 
To see this, by Corollary 5.5. it suffices to check the field of definition for all the distinct representations of the holonomy group in $\mathrm{GL}(2 ; \mathbf{C})$. For most of the groups, every representation will be conjugate to one defined over an imaginary quadratic number field. Both $C_{12}$ and $C_{24}$ can arise via central products of nil 3-manifold groups for which Theorem 6.4 can be applied to show that the resulting $\mathrm{AB}$-groups are arithmetically inadmissible. For $C_{5}$-holonomy, we can apply Proposition 6.1, which in turn yields the result for $C_{10}$-holonomy, since any arithmetic representation for an $\mathrm{AB}$-group with $C_{10}$-holonomy would yield one for an AB-group with $C_{5}$-holonomy.

\section{Final comment}

As discussed in the remark following Corollary 6.5, one cannot expect to obtain manifolds of finite volume with just a single cusp in the above construction. However, a natural question is whether or not one can guarantee that $\mathbf{H}_{\mathbf{C}}^{2} / \Pi$ be constructed to be a manifold. For instance, in the second family (2), the only congruence subgroup which contains $\Gamma$ is the level two congruence subgroup. This is seen to have torsion though, as

$$
\left(\begin{array}{ccc}
-1 & 0 & 0 \\
0 & 1 & 0 \\
0 & 0 & 1
\end{array}\right)
$$

is an element of this subgroup. However by results of Borel [3], we can guarantee that the first and third families map into torsion free groups. We can simply conjugate by a dilation to ensure that the representation maps into a congruence subgroup which is torsion free. [3] is needed in showing that the congruence subgroup is torsion free.

\section{Appendix: solutions to Equation (1)}

Here, we include the matrices found by solving Equation (11) in $\S 7$. For all possible faithful representations, see [24].

(1)

$$
\begin{array}{rlr}
a & =\left(\begin{array}{ccc}
1 & 2 k & 2 k \\
-2 k & 1-2 k^{2} & -2 k^{2} \\
2 k & 2 k^{2} & 1+2 k^{2}
\end{array}\right), & b=\left(\begin{array}{ccc}
1 & 2 k i & 2 k i \\
2 k i & 1-2 k^{2} & 2 k^{2} \\
-2 k i & 2 k^{2} & 1+2 k^{2}
\end{array}\right) \\
c & =\left(\begin{array}{ccc}
1 & 0 & 0 \\
0 & 1+8 k i & 8 k i \\
0 & -8 k i & 1-8 k i
\end{array}\right) .
\end{array}
$$

Algebraic 83 Geometric Topology, Volume 4 (2004) 
(2)

$$
\begin{array}{rlrl}
a & =\left(\begin{array}{ccc}
1 & 2 k & 2 k \\
-2 k & 1-2 k^{2} & -2 k^{2} \\
2 k & 2 k^{2} & 1+2 k^{2}
\end{array}\right), & b=\left(\begin{array}{ccc}
1 & 2 k i & 2 k i \\
2 k i & 1-2 k^{2} & 2 k^{2} \\
-2 k i & 2 k^{2} & 1+2 k^{2}
\end{array}\right), \\
c=\left(\begin{array}{ccc}
1 & 0 & 0 \\
0 & 1+8 k i & 8 k i \\
0 & -8 k i & 1-8 k i
\end{array}\right), & \alpha=\left(\begin{array}{ccc}
-1 & 0 & 0 \\
0 & 1+k i & k i \\
0 & -k i & 1-k i
\end{array}\right) .
\end{array}
$$

(3)

$$
\begin{aligned}
a & =\left(\begin{array}{ccc}
1 & 4 k & 4 k \\
-4 k & 1-8 k^{2} & -8 k^{2} \\
4 k & 8 k^{2} & 1+8 k^{2}
\end{array}\right), & b & =\left(\begin{array}{ccc}
1 & 4 k i & 4 k i \\
4 k i & 1-8 k^{2} & 8 k^{2} \\
-4 k i & 8 k^{2} & 1+8 k^{2}
\end{array}\right), \\
c= & \left(\begin{array}{ccc}
1 & 0 & 0 \\
0 & 1+16 k i & 16 k i \\
0 & -16 k i & 1-16 k i
\end{array}\right), & \alpha & =\left(\begin{array}{ccc}
1 & 2 k & 2 k \\
2 k & 1-2 k^{2} & -2 k^{2} \\
-2 k & 2 k^{2} & 1+2 k^{2}
\end{array}\right) \iota
\end{aligned}
$$

(4)

$$
\begin{aligned}
& a=\left(\begin{array}{ccc}
1 & 4 k & 4 k \\
-4 k & 1-8 k^{2} & -8 k^{2} \\
4 k & 8 k^{2} & 1+8 k^{2}
\end{array}\right), \quad b=\left(\begin{array}{ccc}
1 & 4 k i & 4 k i \\
4 k i & 1-8 k^{2} & 8 k^{2} \\
-4 k i & 8 k^{2} & 1+8 k^{2}
\end{array}\right) \text {, } \\
& c=\left(\begin{array}{ccc}
1 & 0 & 0 \\
0 & 1+16 k i & 16 k i \\
0 & -16 k i & 1-16 k i
\end{array}\right), \quad \alpha=\left(\begin{array}{ccc}
-1 & 2 k+2 k i & 2 k+2 k i \\
-2 k+2 k i & 1-4 k^{2} & -4 k^{2} \\
2 k-2 k i & 4 k^{2} & 1+4 k^{2}
\end{array}\right) \text {, } \\
& \beta=\left(\begin{array}{ccc}
1 & 2 k & 2 k \\
-2 k & 1-4 k^{2} & -4 k^{2} \\
2 k & 4 k^{2} & 1+4 k^{2}
\end{array}\right) \iota \text {. }
\end{aligned}
$$

(5)

$$
\begin{array}{rlrl}
a & =\left(\begin{array}{ccc}
1 & 2 k & 2 k \\
-2 k & 1-2 k^{2} & -2 k^{2} \\
2 k & 2 k^{2} & 1+2 k^{2}
\end{array}\right), & b=\left(\begin{array}{ccc}
1 & 2 k i & 2 k i \\
2 k i & 1-2 k^{2} & 2 k^{2} \\
-2 k i & 2 k^{2} & 1+2 k^{2}
\end{array}\right), \\
c=\left(\begin{array}{ccc}
1 & 0 & 0 \\
0 & 1+8 k i & 8 k i \\
0 & -8 k i & 1-8 k i
\end{array}\right), & \alpha=\left(\begin{array}{ccc}
i & 0 & 0 \\
0 & 1+\frac{p k}{2} i & \frac{p k}{2} i \\
0 & -\frac{p k}{2} i & 1-\frac{p k}{2} i
\end{array}\right) .
\end{array}
$$

Algebraic 85 Geometric Topology, Volume 4 (2004) 
(6)

$$
\begin{aligned}
& a=\left(\begin{array}{ccc}
1 & 24 k & 24 k \\
-24 k & 1-288 k^{2} & -288 k^{2} \\
24 k & 288 k^{2} & 1+288 k^{2}
\end{array}\right) \\
& b=\left(\begin{array}{ccc}
1 & -12+12 \sqrt{3} i & -12+12 \sqrt{3} i \\
12+12 \sqrt{3} i & 1-288 k^{2} & -288 k^{2} \\
-12-12 \sqrt{3} i & 288 k^{2} & 1+288 k^{2}
\end{array}\right), \\
& c=\left(\begin{array}{ccc}
1 & 0 & 0 \\
0 & 1+144 k \sqrt{3} i & 144 k \sqrt{3} i \\
0 & -144 k \sqrt{3} i & 1-144 k \sqrt{3} i
\end{array}\right) \text {, } \\
& \alpha=\left(\begin{array}{ccc}
1 & \mu & \mu \\
6\left[k+2 k_{1}\right]+6 \sqrt{3}\left[k-2 k_{1}\right] i & 1-\sigma & -\sigma \\
-6\left[k+2 k_{1}\right]-6 \sqrt{3}\left[k-2 k_{1}\right] i & \sigma & 1+\sigma
\end{array}\right)\left(\begin{array}{ccc}
\zeta_{3} & 0 & 0 \\
0 & 1 & 0 \\
0 & 0 & 1
\end{array}\right) \text {, }
\end{aligned}
$$

where

$$
\sigma=\frac{1}{2}\left[36\left(k+2 k_{1}\right)^{2}+108\left(k-2 k_{1}\right)^{2}-192 k \sqrt{3}\left(k\|z\|^{2}+2 k_{2}\right) i\right]
$$

and

(7)

$$
\mu=-6\left[k+2 k_{1}\right]+6 \sqrt{3}\left[k-2 k_{1}\right] i
$$

$$
\begin{aligned}
a & =\left(\begin{array}{ccc}
1 & 12 k & 12 k \\
-12 k & 1-144 k^{2} & -144 k^{2} \\
12 k & 144 k^{2} & 1+144 k^{2}
\end{array}\right), \\
b & =\left(\begin{array}{ccc}
6 k+6 k \sqrt{3} i & 1-144 k^{2} & -144 k^{2} \\
6 k & -6 k+6 k \sqrt{3} i & -6 k+6 k \sqrt{3} i \\
-6 k-6 k \sqrt{3} i & 144 k^{2} & 1+144 k^{2}
\end{array}\right), \\
c & =\left(\begin{array}{ccc}
1 & 0 & 0 \\
0 & 1+288 k \sqrt{3} i & 288 k \sqrt{3} i \\
0 & -288 k \sqrt{3} i & 1-288 \sqrt{3} i
\end{array}\right), \\
\alpha & =\left(\begin{array}{ccc}
3 k-3 k \sqrt{3} i & 1-\chi & -6 k \\
-3 k+3 k \sqrt{3} i & \chi & 1+\chi
\end{array}\right)\left(\begin{array}{ccc}
\zeta_{6} & 0 & 0 \\
0 & 1 & 0 \\
0 & 0 & 1
\end{array}\right),
\end{aligned}
$$

where

$$
\chi=-36 k^{2}+12 k\left(4 k_{1}+3 k\right) \sqrt{3} i
$$

\section{References}

[1] D Allcock, Reflection groups on the octave hyperbolic plane, J. Algebra 213 (1999) 467-498 MathReview

[2] N Bergeron, Premier nombre de Betti et spectre du laplacien de certaines variétés hyperboliques, Enseign. Math. 46 (2000) 109-137 MathReview 
[3] A Borel, Compact Clifford-Klein forms of symmetric spaces, Topology 2 (1963) 111-122 MathReview

[4] A Borel, Linear algebraic groups, second edition, Graduate Texts in Mathematics 126, Springer-Verlag, New York (1991) MathReview

[5] MR Bridson, A Haefliger, Metric spaces of non-positive curvature, Grundlehren der Mathematischen Wissenschaften 319, Springer-Verlag, Berlin (1999) MathReview

[6] J S Chahal, Solution of the congruence subgroup problem for solvable algebraic groups, Nagoya Math. J. 79 (1980) 141-144 MathReview

[7] K Corlette, Archimedean superrigidity and hyperbolic geometry, Ann. of Math. 135 (1992) 165-182 MathReview

[8] C W Curtis, I Reiner, Representation theory of finite groups and associative algebras, Reprint of the 1962 original, Wiley Classics Library, John Wiley \& Sons Inc. New York (1988) MathReview

[9] K Dekimpe, Almost-Bieberbach groups: affine and polynomial structures, Lecture Notes in Mathematics 1639, Springer-Verlag, Berlin (1996) MathReview

[10] K Dekimpe, B Eick, Computational aspects of group extensions and their applications in topology, Experiment. Math. 11 (2002) 183-200 MathReview

[11] F T Farrell, S Zdravkovska, Do almost flat manifolds bound?, Michigan Math. J. 30 (1983) 199-208 MathReview

[12] W M Goldman, Complex hyperbolic geometry, Oxford Mathematical Monographs, The Clarendon Press, Oxford University Press, New York (1999) MathReview

[13] M Gromov, Almost flat manifolds, J. Differential Geom. 13 (1978) 231-241 MathReview

[14] E Hamilton, Abelian subgroup separability of Haken 3-manifolds and closed hyperbolic $n$-orbifolds, Proc. London Math. Soc. 83 (2001) 626-646 MathReview

[15] G C Hamrick, D C Royster, Flat Riemannian manifolds are boundaries, Invent. Math. 66 (1982) 405-413 MathReview

[16] Y Kamishima, Cusp cross-sections of hyperbolic orbifolds by nilmanifolds, in preparation

[17] I Kim, J R Parker, Geometry of quaternionic hyperbolic manifolds, Math. Proc. Cambridge Philos. Soc. 135 (2003) 291-320 MathReview

[18] D D Long, Immersions and embeddings of totally geodesic surfaces, Bull. London Math. Soc. 19 (1987) 481-484 MathReview

[19] D D Long, A W Reid, On the geometric boundaries of hyperbolic 4-manifolds, Geom. Topol. 4 (2000) 171-178 MathReview

[20] D D Long, A W Reid, All flat manifolds are cusps of hyperbolic orbifolds, Algebr. Geom. Topol. 2 (2002) 285-296 MathReview 
[21] C Maclachlan, A W Reid, The arithmetic of hyperbolic 3-manifolds, Graduate Texts in Mathematics 219, Springer-Verlag, New York (2003) MathReview

[22] A I Mal'cev, On homomorphisms onto finite groups, Ivanov. Gos. Ped. Inst. Ucen. Zap. 18 (1958) 49-60

[23] G A Margulis, G A Soŭfer, Maximal subgroups of infinite index in finitely generated linear groups, J. Algebra 69 (1981) 1-23 MathReview

[24] D B McReynolds, Ph.D. Thesis (In preparation)

[25] MQ Ouyang, Geometric invariants for Seifert fibred 3-manifolds, Trans. Amer. Math. Soc. 346 (1994) 641-659 MathReview

[26] V Platonov, A Rapinchuk, Algebraic groups and number theory, translated from the 1991 Russian original by Rachel Rowen, Pure and Applied Mathematics 139, Academic Press, Boston, MA (1994) MathReview

[27] MS Raghunathan, Discrete subgroups of Lie groups, Ergebnisse series 68, Springer-Verlag, New York (1972), MathReview

[28] J G Ratcliffe, Foundations of hyperbolic manifolds, Graduate Texts in Mathematics 149, Springer-Verlag, New York (1994) MathReview

[29] P Scott, The geometries of 3-manifolds, Bull. London Math. Soc. 15 (1983) 401-487 MathReview

[30] J-P Serre, Linear representations of finite groups, translated from the second French edition by Leonard L. Scott, Graduate Texts in Mathematics 42, Springer-Verlag, New York (1977) MathReview

[31] W P Thurston, Three-dimensional geometry and topology. Vol. 1, edited by Silvio Levy, Princeton Mathematical Series 35, Princeton University Press, Princeton, NJ (1997) MathReview

[32] S Upadhyay, A bounding question for almost flat manifolds, Trans. Amer. Math. Soc. 353 (2001) 963-972 MathReview

[33] M-F Vignéras, Arithmétique des algèbres de quaternions, volume 800 of Lecture Notes in Mathematics, Springer-Verlag, Berlin (1980)

[34] B A F Wehrfritz, Infinite linear groups. An account of the group-theoretic properties of infinite groups of matrices, Ergebnisse series 76, Springer-Verlag, New York (1973) MathReview

[35] A Weil, Algebras with involutions and the classical groups, J. Indian Math. Soc. (N.S.) 24 (1960) 589-623 (1961) MathReview

[36] A Weil, Basic number theory, reprint of the second (1973) edition, Classics in Mathematics, Springer-Verlag, Berlin (1995) MathReview

University of Texas, Austin, TX 78712, USA

Email: dmcreyn@math.utexas.edu

Received: 2 April 2004 Revised: 24 August 04 